\title{
The Delivery of Water During Terrestrial Planet Formation
}

\author{
David P. O'Brien ${ }^{1}$ • Andre Izidoro ${ }^{2,3}$. \\ Seth A. Jacobson ${ }^{4,5,6}$ - Sean N. Raymond ${ }^{3}$. \\ David C. Rubie ${ }^{5}$
}

Received: 12 September 2016 / Accepted: 17 January 2018 / Published online: 8 February 2018

(C) Springer Science+Business Media B.V., part of Springer Nature 2018

\begin{abstract}
The planetary building blocks that formed in the terrestrial planet region were likely very dry, yet water is comparatively abundant on Earth. Here we review the various mechanisms proposed for the origin of water on the terrestrial planets. Various in-situ mechanisms have been suggested, which allow for the incorporation of water into the local planetesimals in the terrestrial planet region or into the planets themselves from local sources, although all of those mechanisms have difficulties. Comets have also been proposed as a source, although there may be problems fitting isotopic constraints, and the delivery efficiency is very low, such that it may be difficult to deliver even a single Earth ocean of water this way. The most promising route for water delivery is the accretion of material from beyond the snow line, similar to carbonaceous chondrites, that is scattered into the terrestrial planet region as the planets are growing. Two main scenarios are discussed in detail. First is the classical scenario in which the giant planets begin roughly in their final locations and the disk of planetesimals and embryos in the terrestrial planet region extends all the way into the outer asteroid belt region. Second is the Grand Tack scenario, where early inward and outward migration of the giant planets implants material from beyond the snow line into the asteroid belt and terrestrial planet region, where it can be accreted by the growing planets.
\end{abstract}

The Delivery of Water to Protoplanets, Planets and Satellites

Edited by Michel Blanc, Allessandro Morbidelli, Yann Alibert, Lindy Elkins-Tanton, Paul Estrada,

Keiko Hamano, Helmut Lammer, Sean Raymond and Maria Schönbächler

D.P. O’Brien

obrien@psi.edu

1 Planetary Science Institute, 1700 E. Ft. Lowell, Suite 106, Tucson, AZ 85719, USA

2 Present address: Grupo de Dinamica Orbital Planetologia, UNESP, Universidade Estadual Paulista, Guaratingueta, CEP 12.516-410, Sao Paulo, Brazil

3 Laboratoire d'Astrophysique de Bordeaux, CNRS, Universite de Bordeaux, B18N, Allee Geoffroy Saint-Hilaire, 33615 Pessac, France

4 Present address: Department of Earth and Planetary Sciences, Northwestern University, Evanston, IL 60208, USA

5 Bayerisches Geoinstitut, University of Bayreuth, 95490 Bayreuth, Germany

6 Observatoire de la Côte d'Azur, Bd. de l'Observatoire, CS 34229, 06304, Nice Cedex 4, France 
Sufficient water is delivered to the terrestrial planets in both scenarios. While the Grand Tack scenario provides a better fit to most constraints, namely the small mass of Mars, planets may form too fast in the nominal case discussed here. This discrepancy may be reduced as a wider range of initial conditions is explored. Finally, we discuss several more recent models that may have important implications for water delivery to the terrestrial planets.

Keywords Terrestrial planet formation · Water delivery

\section{Introduction}

The existence of water on the Earth is somewhat of a puzzle. Evidence suggests that solids that condensed around $1 \mathrm{AU}$ in the solar nebula were dry and highly reduced, yet Earth clearly has water on its surface, and likely even more in its mantle. Other terrestrial planets show evidence for water as well. The large D/H ratio of Venus' atmosphere suggests an ancient ocean was lost to space (Donahue et al. 1982), while geomorphology (Baker 2007), neutron spectroscopy data (Feldman et al. 2004), and various other lines of evidence point to a wet past for Mars and significant water still present as subsurface ice. Numerous mechanisms have been suggested for delivering this water to the terrestrial planets, or allowing for incorporation from local sources. In this chapter, we review the different mechanisms and assess their plausibility, in light of available constraints and evidence.

We begin the chapter with an overview of the key geochemical and cosmochemical evidence for the origin of Earth's water in Sect. 2. In Sect. 3 we describe the different stages of terrestrial planet formation, from the growth of planetesimals and planetary embryos through the final stage of accretion, and discuss two different scenarios for late-stage accretion. We first describe the 'classical' scenario, with a disk of planetesimals and embryos extending from the terrestrial planet region into the outer asteroid belt region, and the giant planets in roughly their current locations (e.g. Chambers 2001; Raymond et al. 2004; O’Brien et al. 2006; Raymond et al. 2009; Izidoro et al. 2013, 2014). Second, we discuss the 'Grand Tack' scenario of Walsh et al. (2011), where the giant planets experience an inwards-then-outward migration that implants material from beyond the snow line into the asteroid belt and terrestrial planet region.

Beginning our discussion of water delivery, we first describe various 'in-situ' mechanisms in Sect. 4 that could allow for the incorporation of water into planetesimals local to the growing terrestrial planets or directly into the planets themselves. We then discuss the possibility of water delivery by comets in Sect. 5. Problems and difficulties with both scenarios are discussed. Finally, in Sect. 6 we discuss water delivery in the classical and Grand Tack scenarios of late-stage planet formation. Both scenarios allow for sufficient water delivery to explain the water budget of Earth. The Grand Tack scenario provides the best fit to most constraints, namely the small mass of Mars, but some issues with the timescale of planet formation remain. We summarize our discussion and point to further avenues of research on this topic in Sect. 7.

\section{Geochemical and Cosmochemical Constraints}

Here we discuss some of the key constraints on modeling of water delivery to the terrestrial planets, with a focus on the Earth. For further details see the other chapters in this collection 


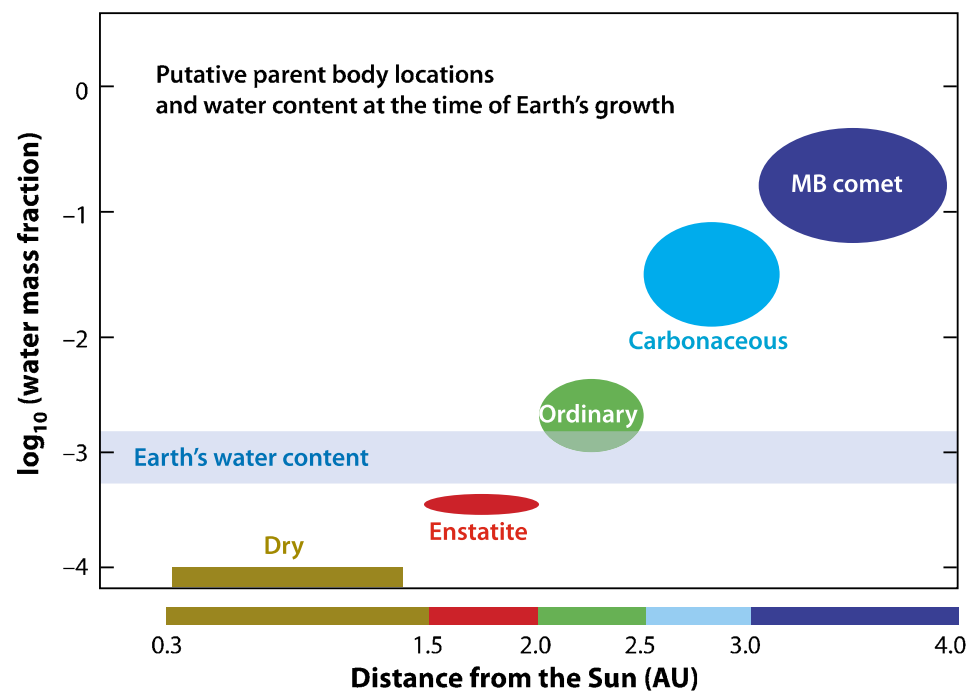

Fig. 1 Water contents of material estimated to have formed at different heliocentric distances, with the range of Earth's possible water content shown for comparison. Ordinary chondrites are spectroscopically linked to S-type asteroids, which dominate the inner asteroid belt, while carbonaceous chondrites are thought to originate from C-type asteroids, which dominate the outer belt. There is not a clear spectral link between enstatite chondrites and a known class of asteroids, although their chemistry suggests that they formed interior to the asteroid belt. Figure from Morbidelli et al. (2012b)

by Peslier et al. (2017) on water in the Earth and Alexander et al. (2018) on water in small Solar System bodies.

Lécuyer (1998) estimate the total mass of water in the Earth's crust, oceans, and atmosphere to be $2.8 \times 10^{-4} \mathrm{M}_{\oplus}$. The water content of the mantle is more uncertain, with estimates ranging from $0.8-8 \times 10^{-4} \mathrm{M}_{\oplus}$ (Lécuyer 1998) to $2 \times 10^{-3} \mathrm{M}_{\oplus}$ (Marty 2012). The primitive mantle could have potentially contained even more, as much as 10-50 Earth oceans (Dreibus and Waenke 1989; Righter and Drake 1999; Abe et al. 2000), although there is no definitive constraint on this value. The core may also contain significant water, although that too is unclear. Nomura et al. (2014) estimate a quantity of hydrogen in the core equivalent to 80 Earth oceans of water, whereas Badro et al. (2014) find very little. From this, a reasonable lower limit for the amount of water that must be delivered to (and retained by) the Earth is $5 \times 10^{-4} \mathrm{M}_{\oplus}$, although the actual amount of water delivered could be significantly larger.

It has traditionally been assumed that the solar nebula around $1 \mathrm{AU}$ was too hot for ice to condense and be incorporated into local Earth-forming material. This is based in part on associations between different meteorite types and their asteroidal parent bodies or estimated locations of formation (Fig. 1), showing a trend towards extremely dry material near Earth's location. However, nebula models taking into account stellar irradiation, viscous heating, and time-dependent opacities (e.g. Hueso and Guillot 2005; Davis 2005; Garaud and Lin 2007; Oka et al. 2011; Bitsch et al. 2015; Baillié et al. 2015) show that the ice-condensation line (the snowline) can move inwards to $\sim 1 \mathrm{AU}$ as the accretion of gas onto the central star slows. Morbidelli et al. (2016) show that even if the temperature around 1 AU is cold enough for ice to condense, the snowline could be fossilized at around 3 AU since the gas drifts inwards more quickly than the snowline moves, and the formation of a proto-Jupiter 
would block the migration of icy particles into the inner disk. Thus, there is still a problem of condensing ice and incorporating it into planetesimals in the terrestrial planet zone.

Water is clearly present in bodies in the outer asteroid belt and beyond, however. Primitive meteorites such as CM and CI chondrites can have $~ 5-20 \%$ water by mass, and are believed to come from asteroids such as the C-types that dominate the outer asteroid belt (Burbine et al. 2002). Ceres at $\sim 2.7$ AU contains several tens of percent water ice (e.g. McCord and Sotin 2005) and shows signs of water vapor emission (Küppers et al. 2014) and recent aqueous activity (de Sanctis et al. 2016). Water ice has been directly detected on the asteroid Themis at 3.1 AU (Campins et al. 2010; Rivkin and Emery 2010). Some outer asteroid belt bodies called main-belt comets, which show signs of activity, likely have at least $10 \%$ water ice (Jewitt 2012). Centaurs, comets, trans-Neptunian objects, and many outer planet satellites are also ice-rich.

A key constraint on the origin of Earth's water is the deuterium/hydrogen $(\mathrm{D} / \mathrm{H}) \mathrm{ratio}$, estimated by Lécuyer (1998) to be $\sim 150 \times 10^{-6}$, or about $6 \times$ the solar value. This value is close to the mean whole-rock value for water-rich carbonaceous chondrite meteorites (e.g. Dauphas et al. 2000; Robert 2003, 2006; Alexander et al. 2012), suggesting that material similar to carbonaceous chondrites is a potential source of Earth's water. The value for comets is often quoted as being $12 \times$ the solar value, or $2 \times$ terrestrial, although there are some exceptions to this, as described further in Sect. 5. While carbonaceous chondrites may provide a good match to the Earth's D/H ratio, their oxygen isotope ratios and volatile element compositions, when compared to the Earth, suggest that carbonaceous chondritic material may have only contributed $\sim 2 \%$ of the Earth's mass (Drake and Righter 2002; Marty 2012).

There are geochemical constraints on the timing of water delivery as well. Studies of core formation in the Earth suggest that the early material that accreted to form Earth must have been highly reduced (Wood et al. 2008; Rubie et al. 2011), which would preclude the presence or delivery of significant water early on. As we discuss in Sect. 6, Rubie et al. (2015b) combined a core-mantle differentiation model with N-body simulations to explore this issue in further detail.

Some models (e.g. Rubie et al. 2004; Siebert et al. 2013) have proposed that core-mantle differentiation occurs under oxidizing conditions, which could be consistent with abundant water being accreted to Earth very early. In those models, accreted silicate material has a high $\mathrm{FeO}$ content (e.g. $\mathrm{FeO}$ mole fraction $X_{\mathrm{FeO}} \approx 0.2$ ). During core formation, oxygen partitions into the core (as $\mathrm{FeO}$ ) and reduces $X_{\mathrm{FeO}}$ of the mantle to the observed value of $\sim 0.06$. However, there is a significant problem with such models because several weight percent $\mathrm{Si}$ also partitions into the core, and every mole of Si that enters the core causes two moles of $\mathrm{FeO}$ to be transferred to the mantle. This makes it impossible for the silicate mantle to achieve the observed low final $X_{\mathrm{FeO}}$ value (Rubie et al. 2015b,a).

\section{Stages of Terrestrial Planet Formation}

The process of terrestrial planet formation begins with the condensation of the first solids 4.567-4.568 Gyr ago (e.g. Amelin et al. 2002; Bouvier and Wadhwa 2010; Connelly et al. 2012) and can be considered complete by the end of the heavy bombardment of the inner Solar System around 4.1-3.8 Gyr ago (Tera et al. 1974; Chapman et al. 2007; Bottke et al. 2012; Marchi et al. 2012; Morbidelli et al. 2012a). The gaseous solar nebula dissipated within $\sim 2-10 \mathrm{Myr}$ of the formation of the first solids (e.g. Haisch et al. 2001; Kita et al. 2005), and during that time, the initial planetesimals and planetary embryos formed 
and the giant planets grew their cores and captured their gaseous envelopes. While Mars may essentially be a leftover embryo from this early phase (Dauphas and Pourmand 2011), the larger terrestrial planets experienced a much more drawn-out accretion process, lasting around 30-100 Myr (Raymond et al. 2006, 2009; Bromley and Kenyon 2006; O'Brien et al. 2006; Touboul et al. 2007; Kleine et al. 2009; Morbidelli et al. 2012b). For the Earth, the last major impact was likely that which formed the Moon (Cameron 2000; Canup and Asphaug 2001; Canup 2004; Ćuk and Stewart 2012; Canup 2012; Reufer et al. 2012). Here we give a brief overview of the different stages of terrestrial planet formation to provide a background for the rest of the chapter. There have been numerous reviews of terrestrial planet formation over the last decade or so, which the reader can consult for further details (e.g. Chambers 2004; Chambers et al. 2010; Righter and O'Brien 2011; Morbidelli et al. 2012b; Raymond et al. 2014).

The formation of terrestrial planets has often been described as consisting of three main stages, although there may be some overlap between them. The first stage, in which solid grains in the nebula accumulate into kilometer-scale or larger planetesimals is one of the most uncertain. The main problem in this stage is that the radial drift of bodies in the nebula due gas drag effects (e.g. Weidenschilling 1977, 1980) is fastest for meter-scale bodies, which are too large to be coupled to the gas, but small enough in mass that they feel a substantial drag force. Bodies of this size can drift into the Sun on a timescale $\sim 100$ years, and thus any planetesimal growth process must be rapid enough to overcome this meter size barrier.

One proposal is that planetesimals grow by pairwise accretion of smaller grains in the nebula (e.g. Weidenschilling 1980; Weidenschilling and Cuzzi 1993; Weidenschilling et al. 1997; Wurm et al. 2001; Weidenschilling 2011). One potential issue with this scenario is that it requires a non-turbulent nebula, at least at the location and time when planetesimals were forming, which may not necessarily be the case (Armitage 2011). Otherwise, growth is slowed and bodies may not grow past the meter size barrier fast enough, and turbulence may drive collisions and erosion of bodies, rather than growth.

Another scenario is that particles settling to the midplane of the nebula may exceed a critical density such that planetesimals could grow purely by self-gravity, a process called gravitational instability (e.g. Goldreich and Ward 1973; Ward 2000; Youdin and Shu 2002). Like with pairwise accretion, nebular turbulence can impede this process, but even if the nebula is initially non-turbulent, shear between the midplane particle layer and the overlying gas could drive turbulence and prevent the solids from reaching a critical density (e.g. Weidenschilling and Cuzzi 1993; Weidenschilling 2003).

Some models, however, take advantage of turbulence to aid in the formation of planetesimals. In a process called turbulent concentration, eddies in the solar nebula may preferentially concentrate bodies of specific sizes, in particular chondrule-sized bodies, and lead to rapid growth (Cuzzi et al. 2001, 2008; Chambers 2010). Alternatively, meter-scale boulders may become concentrated in over-dense regions of the disk, and the collective drag force on the solids forces the gas to move along with them (rather than sub-Keplerian). This reduces the headwind and radial drift in a process called the streaming instability (Youdin and Goodman 2005; Johansen et al. 2007), and solids drifting into this region continue to accumulate until a critical density is reached.

Regardless of the specific process by which planetesimals form, they soon enter a second stage of growth in which they begin to gravitationally accrete one-another and grow into larger planetary embryos. At first, this proceeds through a process called runaway accretion (e.g. Greenberg et al. 1978; Wetherill and Stewart 1989; Kokubo and Ida 1996; Weidenschilling et al. 1997), in which bodies that first manage to grow larger are able to accrete 

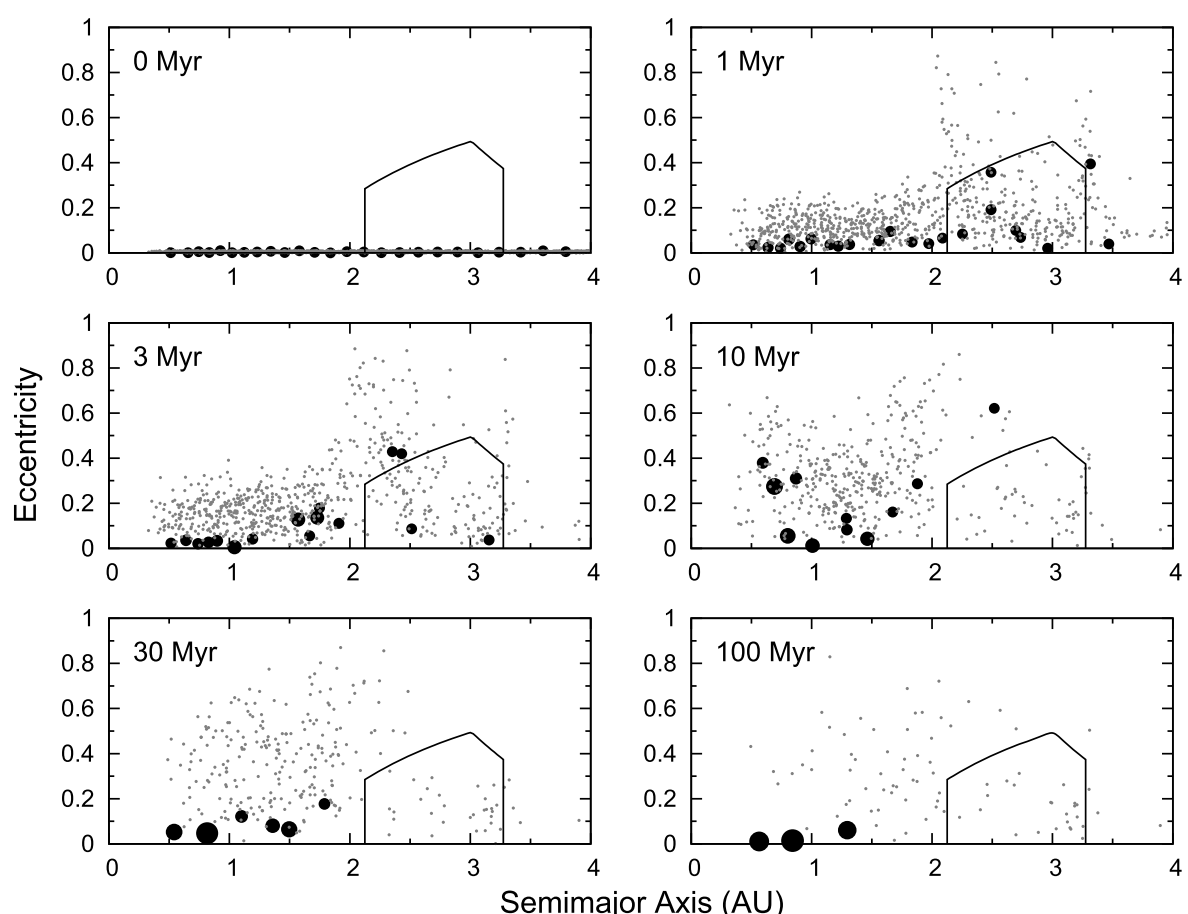

Fig. 2 Terrestrial planet formation from the EJS1 simulation of O'Brien et al. (2006). Large black dots are Mars-mass embryos, and small grey dots are planetesimal with 1/40 the mass of the embryos. The outlined region from $\sim 2-3 \mathrm{AU}$ is the asteroid belt. Figure from O'Brien and Sykes (2011)

even more rapidly due to their enhanced cross sections and gravitational focusing. Once the growing embryos are large enough to significantly excite the relative velocities of surrounding planetesimals, their accretion rate slows and they enter a phase of oligarchic growth in which other, smaller embryos are able to catch up (Kokubo and Ida 1998, 2000). The end result of this stage is a set of Lunar- to Mars-mass embryos embedded in a swarm of remnant planetesimals.

Recently, pebble accretion has emerged as an extremely efficient process for the growth of planetary embryos and giant planet cores (e.g. Ormel and Klahr 2010; Lambrechts and Johansen 2012, 2014; Morbidelli and Nesvorny 2012). So-called pebbles, defined as having a stopping time due to gas drag on the order of their orbital period (generally $\mathrm{mm}$ - to cmscale), are efficiently accreted onto large planetesimals due to gas dynamic effects, and those planetesimals can rapidly grow into planetary embryos. Levison et al. (2015) find that the terrestrial planets themselves can grow largely by accreting pebbles. Water delivery has yet to be quantified in such a model. Other models find that pebble accretion, at least in the terrestrial planet region, results in a swarm of embryos and remnant planetesimals much like the end result of runaway and oligarchic growth (Jacobson and Walsh 2015; Morbidelli et al. 2015; Chambers 2016).

In the final stage of terrestrial planet formation, embryos collide violently with oneanother and grow into the final planets on a timescale of $~ 30-100$ Myr. In the classical scenario, embryos and planetesimals are spread through the terrestrial planet region, and likely into the asteroid belt zone, and the giant planets are fully-formed and located at or near their present locations (e.g. Chambers 2001; Raymond et al. 2004; O'Brien et al. 2006; 
Raymond et al. 2009; Fischer and Ciesla 2014). Figure 2 shows the results of a simulation from O'Brien et al. (2006), starting with 25 Mars-mass embryos (large black dots) and 1000 planetesimals, each with 1/40 the mass of the embryos (small grey dots). The gas disk is assumed to have dissipated by this point, so gas effects are not included. While there is no gas to provide a damping effect on the growing planets, the swarm of planetesimals provides a damping effect due to dynamical friction (e.g. Wetherill and Stewart 1993). Terrestrial planets grow on a timescale of $\sim 100 \mathrm{Myr}$, and the asteroid belt region, outlined in the figure, is dynamically excited and depleted by perturbations from the giant planets and the mutual scattering of embryos (Wetherill 1992; Petit et al. 2001; O'Brien et al. 2007). An important aspect of this scenario from the point of view of water delivery is that material from beyond $\sim 2.5 \mathrm{AU}$ can be scattered into the terrestrial planet region and accreted by the growing planets. This will be discussed in further detail in Sect. 6 .

A major problem with this scenario is that the Mars analogues forming around 1.5 AU are typically 5-10 times more massive than Mars itself. This problem of explaining the small mass of Mars was first noted by Wetherill (1991) and persists over a wide range of parameter space, as shown by Raymond et al. (2009), Morishima et al. (2010), and Izidoro et al. (2015). Numerous models have been tested in an attempt to reconcile this issue, as well as to fit the full range of constraints on terrestrial planet formation and Solar System evolution (e.g. Nagasawa et al. 2005; Thommes et al. 2008; Kominami and Ida 2002, 2004; Ogihara et al. 2007; Morishima et al. 2008, 2010; Lykawka and Ito 2013; Izidoro et al. 2014). Often these models have included the effects of a gas disk, which can provide eccentricity and inclination damping as well drive the migration of embryos through a process called Type 1 migration (e.g. Goldreich and Tremaine 1980; Ward 1986). In general, though, such models are still not able to fit the full range of constraints, or require initial conditions such as eccentric giant planet orbits that are not consistent with Solar System formation models. For purposes of discussing water delivery in a classical scenario in Sect. 6, we will use the O'Brien et al. (2006) simulations described in the previous paragraph. For a more thorough review of the other scenarios listed above, see Morbidelli et al. (2012b) and Raymond et al. (2014).

A significant breakthrough in resolving the problem of forming a small Mars came when Hansen (2009) showed that the mass distribution of the terrestrial planets can be reproduced if the initial distribution of material is confined to a narrow annulus between $\sim 0.7-1$ AU. While they did not propose a specific mechanism for achieving this initial distribution, their work led Walsh et al. (2011) to model how the migration of the giant planets in the presence of nebular gas may allow for the truncation of the planetesimal/embryo distribution around 1 AU. ${ }^{1}$ A large planet like Jupiter can open a gap in the nebular disk and migrate inwards via a process called Type 2 migration, where the planet follows the viscous evolution of the disk (Lin and Papaloizou 1986; Kley and Nelson 2012). If a second, smaller planet (e.g. Saturn) is present just exterior to the first planet, however, the profile of the gap and the resulting torque balance changes, and the inward migration can be halted and reversed (e.g. Masset and Snellgrove 2001; Morbidelli and Crida 2007; Pierens and Nelson 2008; Pierens and Raymond 2011; Pierens et al. 2014; Zhang and Zhou 2010; D'Angelo and Marzari 2012). This allows for a scenario in which Jupiter migrates into the terrestrial planet region, Saturn catches up with it, and the two planets open a mutual gap in the disk, reversing the direction of migration and moving Jupiter and Saturn back outwards to near their current locations. Walsh et al. (2011) termed this migration reversal the 'Grand Tack', in analogy with the turning of a sailboat.

\footnotetext{
${ }^{1}$ Several mechanisms have subsequently been proposed to explain the inner edge at $\sim 0.7 \mathrm{AU}$, including a fossilized silicate condensation line (Morbidelli et al. 2016) and the outward migration of Jupiter's core in a model where it initially forms close to the Sun (Raymond et al. 2016).
} 


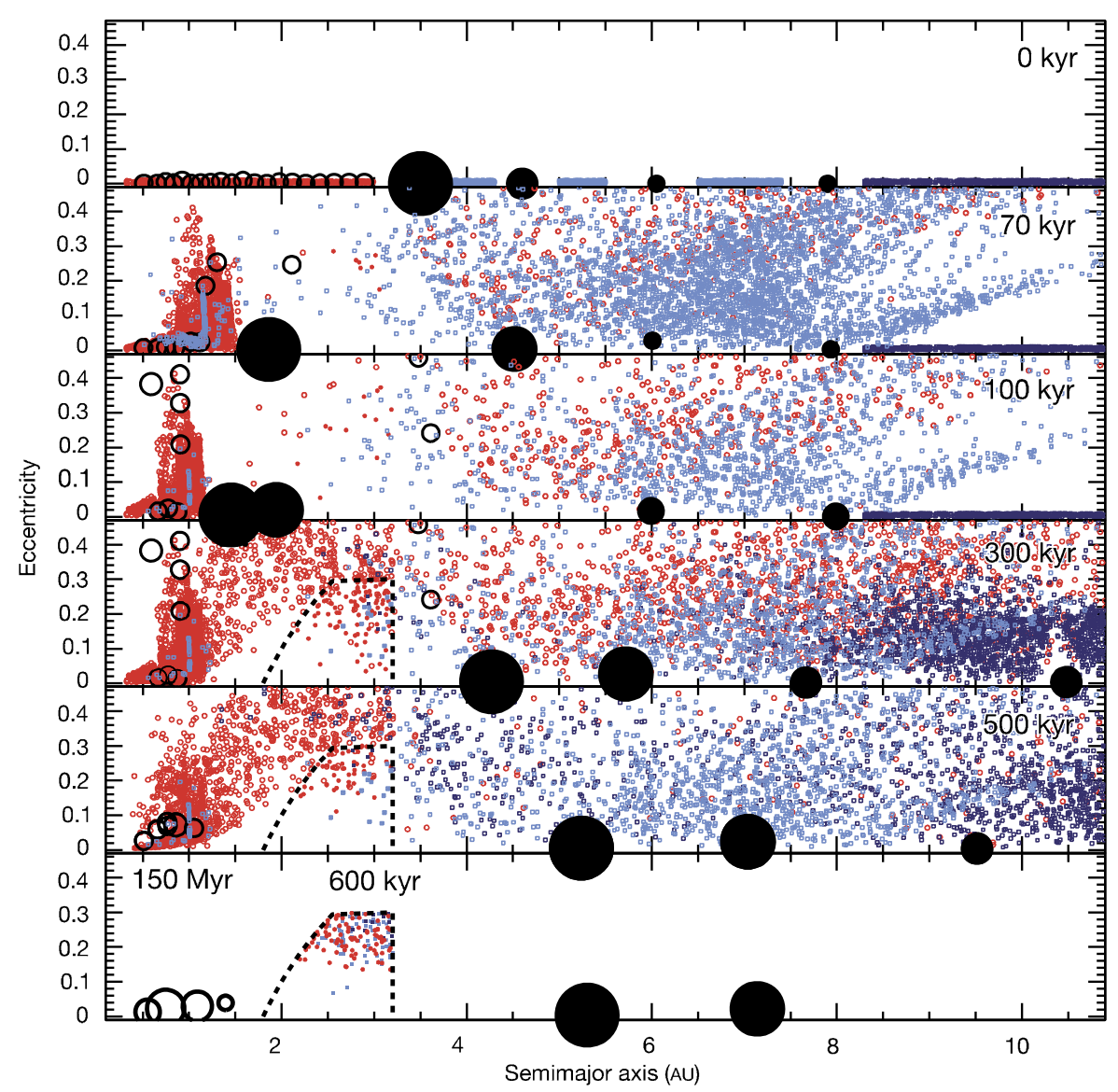

Fig. 3 Figure from Walsh et al. (2011) showing a simulation of the Grand Tack scenario. The top three panels show the inward migration phase of the giant planets (lasting $100 \mathrm{kyr}$ ), and the last three panels show their outward migration (lasting $500 \mathrm{kyr}$ ). The last panel also shows the terrestrial planets formed after $150 \mathrm{Myr}$. Material implanted into the asteroid belt is not uniformly distributed, the inner asteroid belt is dominated by material originating within a few $\mathrm{AU}$ of the Sun (red dots), and the outer belt is dominated by material scattered inwards from the giant planet region (blue dots), which broadly reproduces the S-Type/C-Type dichotomy seen in the asteroid belt today (e.g. Gradie and Tedesco 1982; Mothé-Diniz et al. 2003). The eccentricity distribution of asteroids after $600 \mathrm{kyr}$ is higher than the observed distribution, but Deienno et al. (2016) show that it evolves to roughly match the current distribution over the subsequent 4.5 Gyr of evolution. The terrestrial planets will accrete some of the material scattered inwards from the giant planet region, which is likely water rich. This was quantified in detail by O'Brien et al. (2014)

Walsh et al. (2011) found that if Jupiter reversed direction at 1.5 AU, the inner disk would be truncated at $\sim 1 \mathrm{AU}$, and Mars would generally form from one or a few embryos that scattered outwards and became relatively isolated from the rest of the disk, as in the simulations of Hansen (2009). Furthermore, while the passage of Jupiter through the primordial asteroid belt would be highly disruptive to the initial distribution of material there, Walsh et al. (2011) showed that the scattering effects of Jupiter as it moved inwards and outwards actually repopulated the asteroid belt with original material from the asteroid belt region as well as material scattered inwards from the giant planet region. Figure 3 shows a simulation from Walsh et al. (2011) that illustrates this process, with the top three panels showing the 
inward migration phase (over $100 \mathrm{kyr}$ ), and the last three showing outward migration (over $500 \mathrm{kyr}$ ). The resulting inner asteroid belt is dominated by material originating within a few AU of the Sun (red dots), while the outer belt is dominated by material scattered inwards from the giant planet region (blue dots), broadly reproducing the S-Type/C-Type dichotomy seen in the asteroid belt today (e.g. Gradie and Tedesco 1982; Mothé-Diniz et al. 2003). The eccentricity distribution of asteroids after $600 \mathrm{kyr}$ is higher than the observed distribution, but will evolve to roughly match the current distribution over the subsequent $4.5 \mathrm{Gyr}$ of evolution (Deienno et al. 2016). Material scattered inwards from the giant planet region would likely be water-rich and some would be accreted by the terrestrial planets, which was quantified by O'Brien et al. (2014) and will be discussed in further detail in Sect. 6. Brasser et al. (2016) performed more detailed simulations of the Grand Tack scenario and showed that a reversal of Jupiter at $\sim 2 \mathrm{AU}$ might provide an even better match to some constraints than the 1.5 AU value used by Walsh et al. (2011), although the results in terms of water delivery are expected to be fairly similar.

More recently, there have been several models published besides the Grand Tack that can match many aspects of the inner Solar System. The 'low-mass asteroid belt model' proposes that only a small population of planetesimals formed beyond 1-1.5 AU (Izidoro et al. 2014, 2015; Dra̧żkowska et al. 2016; Morbidelli and Raymond 2016; Raymond and Izidoro 2017b). This is consistent with some models of planetesimal formation by the streaming instability in evolving disks (Drążkowska et al. 2016; Surville et al. 2016). This mass deficit naturally reproduces the large Earth/Mars mass ratio much like Hansen (2009) and the Grand Tack, but requires a mechanism like chaotic excitation to match the asteroid belt (Izidoro et al. 2016). In addition, some models based on pebble accretion can match the terrestrial planets and give a depleted asteroid belt (Levison et al. 2015).

\section{In-Situ/Early Water Delivery}

Numerous studies have shown that water could be incorporated into olivine grains around 1 AU through adsorption directly from the gaseous nebula (Stimpfl et al. 2006; Muralidharan et al. 2008; King et al. 2010; Asaduzzaman et al. 2015). These grains would then follow the steps outlined in Sect. 3 and grow into planetesimals, potentially contributing several oceans worth of water to a growing Earth from local, early-accreted material. Several key questions remain, however. Studies show that the early material that accreted to form Earth was highly reduced (e.g. Wood et al. 2008; Rubie et al. 2011), which would not be the case if it contained significant water. Also, enstatite chondrite meteorites are drier than the Earth (see Fig. 1) and highly reduced, and may be difficult to explain if significant waterbearing material was present. An additional criticism is that this process would lead to the capture of water with a D/H ratio close to Solar, whereas the D/H ratio of the Earth's water is approximately $6 \times$ Solar. Ganguly et al. (2016) show that a fractionation process between adsorbed and nebular water may explain at least part of this enrichment.

Another possibility for the early incorporation of water-bearing material is the oxidation of an early hydrogen atmosphere by $\mathrm{FeO}$ in the terrestrial magma ocean (Ikoma and Genda 2006; Genda and Ikoma 2008). This requires fast growth of the planet in order to capture hydrogen from the solar nebula, which likely dissipated within $10 \mathrm{Myr}$ (Haisch et al. 2001; Kita et al. 2005), in contrast to radiometric estimates for the formation timescale of the Earth that are much longer (e.g. Touboul et al. 2007; Kleine et al. 2009). The D/H ratio of the initial captured gas would be solar, not terrestrial. Hydrodynamic escape of atmosphere over billions of years could potentially lead to a terrestrial D/H ratio (Genda and Ikoma 2008). 
However, this timescale is much longer than the $\sim 100 \mathrm{Myr}$ closure age of the atmosphere estimated from I-Xe isotopes (Wetherill 1975; Avice and Marty 2014).

Even if these two processes are not able to explain all of the Earth's water, or give a terrestrial D/H ratio, they may have still occurred to some degree. Deep-mantle lavas sampling relatively unmixed reservoirs show evidence for solar D/H (Hallis et al. 2015), suggesting that at least some of Earth's water could have been incorporated directly from the solar nebula.

Finally, Ciesla and Lauretta (2005) proposed that water-bearing phyllosilicate dust could drift inwards and be incorporated into planetesimals around $1 \mathrm{AU}$. This would require that water be locked in phyllosilicates while nebular gas was still around. However, many phyllosilicates found in meteorites seem to have been formed by parent-body processes after nebular gas would have dissipated.

\section{Cometary Delivery}

Comets have long been suggested as a source of terrestrial water, given their obvious icerich nature (e.g. Chyba 1987; Delsemme 1992, 1997, 1998; Owen and Bar-Nun 1995). The most common objection to this is that most comets with measured D/H ratios have about twice the terrestrial value. Initially all of those measured comets were Oort-cloud or Halley-type comets. The Jupiter-family comet 103P/Hartley 2 was recently found to have a terrestrial D/H ratio (Hartogh et al. 2011), as was 45P/Honda-Mrkos-Pajdušáková (Lis et al. 2013), hinting that JFCs could be a possible source. However, comet 67P/ChuryumovGerasimenko, also a Jupiter-family comet, was found to have a D/H nearly 3 times terrestrial (Altwegg et al. 2015), so there is no clear trend from the current observational data.

Possibly more problematic than any geochemical constraints, however, is the significant dynamical difficulty in delivering the Earth's water primarily through comets. The probability that comets originating in the giant planet region hit the Earth is $\sim 1 \times 10^{-6}$ (Morbidelli et al. 2000). Various models of outer Solar System evolution suggest that $\sim 30-50 \mathrm{M}_{\oplus}$ of material may have been present in a primordial cometessimal disk (e.g. Malhotra 1993, 1995; Hahn and Malhotra 1999; Gomes et al. 2004, 2005; Tsiganis et al. 2005; Morbidelli et al. 2010). Thus, if $50 \mathrm{M}_{\oplus}$ of comets were initially present and scattered by the giant planets, $5 \times 10^{-5} \mathrm{M}_{\oplus}$ of cometary material would have hit the Earth. As discussed in Sect. 2, the Earth's water content is at least $5 \times 10^{-4} \mathrm{M}_{\oplus}$, so even if those comets were pure ice, only $\sim 10 \%$ of Earth's water could be provided by a cometary source.

\section{Later Delivery}

Morbidelli et al. (2000) and Raymond et al. (2004) showed that if the initial disk of planetesimals and embryos in the inner Solar System extended through the asteroid belt region, water-bearing material from beyond $\sim 2.5 \mathrm{AU}$ can be incorporated into the growing terrestrial planets, often relatively late in the accretion process. O'Brien et al. (2006) performed several sets of simulations with higher resolution that allowed for the analysis of this delivery mechanism in greater detail (see also Raymond et al. 2006, 2007). The O'Brien et al. (2006) simulations started with 25 Mars-mass embryos and 1000 planetesimals, each with $1 / 40$ the mass of an embryo (so that there is equal mass in the planetesimal and embryo populations). Two configurations of Jupiter and Saturn were used: The EJS simulations, with Jupiter and Saturn on their current, eccentric orbits, and the CJS simulations, with Jupiter 
Fig. 4 Relative contributions of material from different semimajor axis zones in the simulations of O'Brien et al. (2006) (adapted from a figure in that paper). The diameter of each symbol is proportional to the diameter of the planet, assuming that they all have the same density. In the EJS simulations, Jupiter and Saturn are on their current, eccentric orbits, and in the CJS simulations, Jupiter and Saturn are on more circular and closely-spaced orbits, as predicted by the Nice Model (Tsiganis et al. 2005; Gomes et al. 2005; Morbidelli et al. 2005)

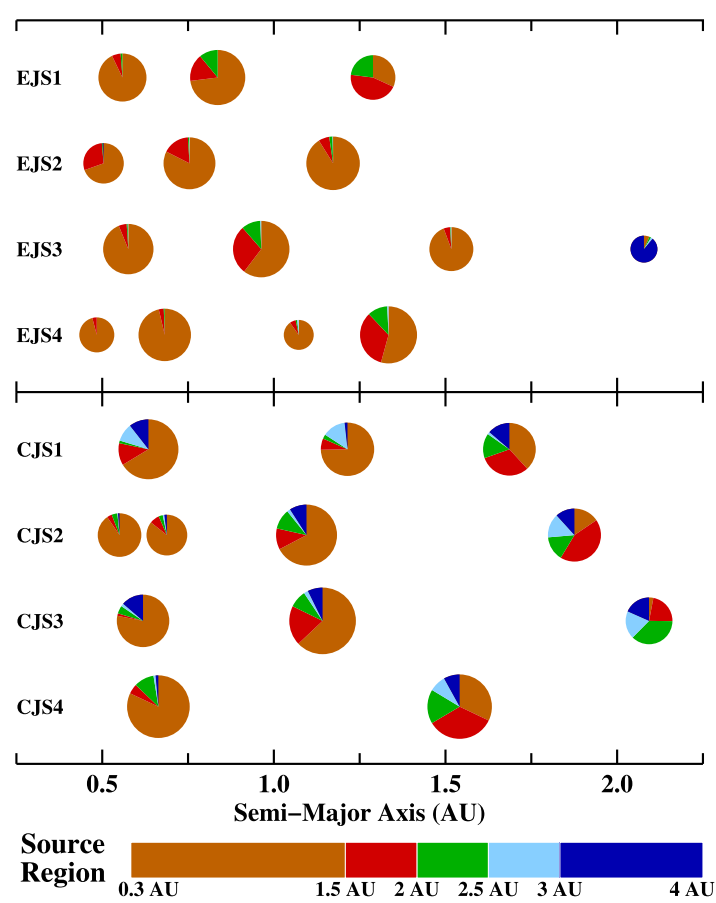

and Saturn on more circular and closely-spaced orbits, as predicted by the Nice Model (Tsiganis et al. 2005; Gomes et al. 2005; Morbidelli et al. 2005). Four simulations were run for each giant planet configuration.

Figure 4 shows the final planets in the simulations of O'Brien et al. (2006), with the contributions of material from different semimajor axis regions. 12 terrestrial planets were formed in the four CJS simulations. All of them accreted at least a few planetesimals from beyond 2.5 AU (median number of 7), and 9 of the planets contained at least one embryo that originated from beyond 2.5 AU. The fraction of each planet's total mass that originates from beyond $2.5 \mathrm{AU}$ ranges from $1.6 \%$ to $38 \%$, with a median value of $15 \%$. On average, about $85 \%$ of the material from beyond $2.5 \mathrm{AU}$ that is accreted by the planets is delivered by embryos.

In contrast, the 14 planets in the EJS simulations receive very little material from beyond 2.5 AU. None of the planets accrete an embryo from beyond 2.5 AU, although one planet (in simulation EJS3) originated as an embryo beyond 2.5 AU and never accreted any other embryos. At most, planets accrete 3 planetesimals from beyond $2.5 \mathrm{AU}$, and 5 of the planets contain no material at all from beyond 2.5 AU. The fraction of each planet's total mass that originates from beyond 2.5 AU (excluding the single-embryo planet in EJS3) has a median value of $0.3 \%$ and ranges from $0 \%$ to $1.6 \%$. This profound disparity between the two sets of simulations is primarily due to the fact that resonances in the asteroid belt region are stronger with Jupiter and Saturn on eccentric orbits, and this leads to most material from beyond 2.5 AU being driven into the Sun or ejected from the Solar System, whereas more of that material slowly diffuses into the terrestrial planet region in the CJS simulations. Interestingly, earlier simulations incorporating only embryos (Morbidelli et al. 2000; Raymond et al. 2004) found that sufficient water could be delivered to the terrestrial planets if Jupiter and Saturn are eccentric. In the O'Brien et al. (2006) simulations, the large number 
of planetesimals tends to aid in the clearing of embryos from the region beyond $2.5 \mathrm{AU}$, such that little or no material from that region ends up being incorporated into the planets when Jupiter and Saturn are eccentric.

The mass fractions of material from beyond 2.5 AU in the final planets can be converted to estimates of water content, for comparison to the Earth. As noted above, the median mass fraction for planets in the CJS simulations is 15\% (ranging from 1.6-38\%). Assuming a mass fraction of $10 \%$ water for the material originating from outside $2.5 \mathrm{AU}$, consistent with the values for carbonaceous chondrites, and also assuming that all accreted volatiles are retained by the planet and not lost in the impact, a $15 \%$ mass fraction implies $0.015 \mathrm{M}_{\oplus}$ of water for an Earth-mass planet, or $30 \times$ the lower limit of $5 \times 10^{-4} \mathrm{M}_{\oplus}$ from Sect. 2. The full range of values for the planets spans $3-75 \times$ the lower limit. Even assuming a more conservative mass fraction of water in the material beyond $2.5 \mathrm{AU}$ of $5 \%$ and assuming that only $10 \%$ of the water is retained in the impact, the median amount of water in an Earth-mass planet is still $1.5 \times$ the lower limit. We note that if the material from beyond $2.5 \mathrm{AU}$ is similar to carbonaceous chondrites, the large amount delivered in these simulations could pose a geochemical conflict. As discussed in Sect. 2, Drake and Righter (2002) and Marty (2012) argue that, based on oxygen isotope ratios and volatile element abundances, carbonaceous chondrites may have only contributed about $2 \%$ of the Earth's mass.

For the EJS simulations, the median mass fraction of material from beyond $2.5 \mathrm{AU}$ that ends up in the final terrestrial planets is $0.3 \%$, with a maximum value of $1.6 \%$. Assuming a $10 \%$ mass fraction of water in the impactors from beyond $2.5 \mathrm{AU}$, and $100 \%$ retention of volatiles in impacts, the median water content for an Earth-mass planet would be $3 \times 10^{-4}$ $\mathrm{M}_{\oplus}$, less than the lower limit value of $5 \times 10^{-4} \mathrm{M}_{\oplus}$. Hence, simulations with an eccentric Jupiter and Saturn have a much harder time forming Earth-like planets, at least in terms of water content.

O'Brien et al. (2006) also analyzed the timing of the delivery of material from beyond 2.5 AU. Figure 5 shows the timing for the planets in the CJS set of simulations. For each planet, the large black dots show the planet mass following the accretion of any embryos from beyond 2.5 AU, and the vertical bars show the planet mass when it has accreted $50 \%$ of its final number of planetesimals originating from beyond 2.5 AU. Nearly all of the embryo impacts occur well after the planet is half grown. In fact, 8 of the 9 planets that do experience at least one impact by an embryo from beyond $2.5 \mathrm{AU}$ actually have that embryo as their final large impactor. Similarly, for the majority of planets, $50 \%$ of their final number of planetesimals from beyond 2.5 AU are not delivered until after the planet has grown to half its size. So whether from embryos or planetesimals, the delivery of potential volatilecarrying material tends to occur late in a planet's growth, and volatile-carrying embryos often occur as the final large impactor. Given the fact that the planets in the EJS simulations contain no embryos from beyond 2.5 AU and at most three such planetesimals, a similar plot can not be constructed for the EJS simulations.

Having water delivered relatively late in the accretion process makes it more likely to be retained, and is also consistent with the findings of Wood et al. (2008) and Rubie et al. (2011) that early material accreting to form Earth must have been highly reduced and the oxidation state increased with time. We stress, though, that while water is delivered relatively late in these simulations, it is not delivered as a late veneer, which is generally considered to be the mass delivered after differentiation has ceased. Differentiation and core formation would almost entirely remove highly siderophile elements from the mantle, and the fact that some do exist today suggests that $\lesssim 1 \%$ of Earth's total mass was accreted after differentiation ceased (Drake and Righter 2002). O'Brien et al. (2006) assumed that differentiation occurs as long as embryo impacts are occurring, and calculated the late veneer mass as that arriving 
Relative Delivery Timescales of Bodies from Beyond 2.5 AU (CJS Simulations)

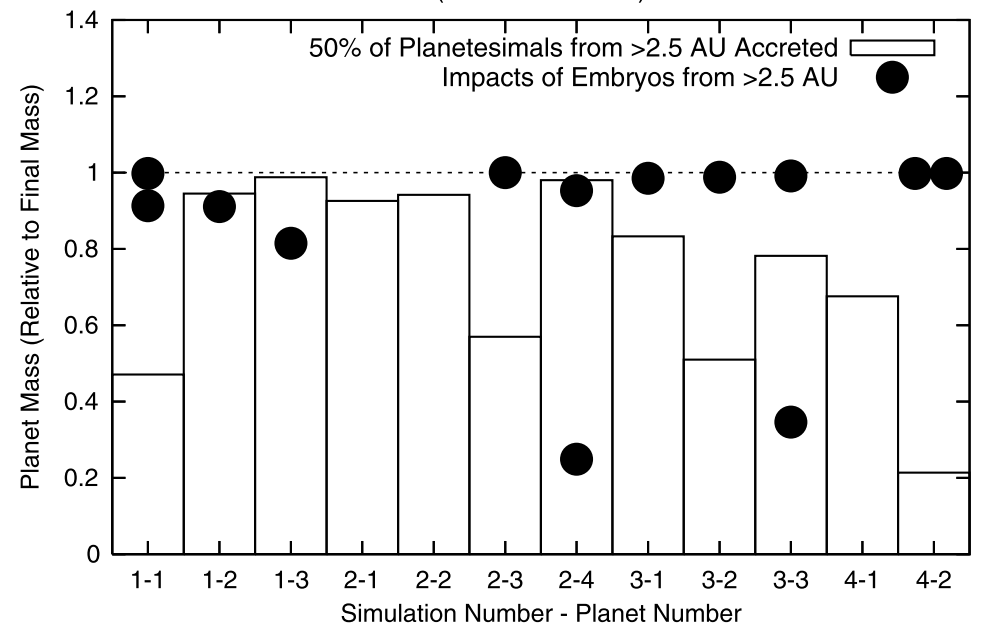

Fig. 5 Timing of the accretion of material from beyond 2.5 AU, which likely contains water and other volatiles, in the CJS simulations of O'Brien et al. (2006). Each column is for a given planet in a given simulation. The bars show the mass of each planet at the point when it has accreted $50 \%$ of its final number of planetesimals originating from beyond $2.5 \mathrm{AU}$. Almost all planets reach the $50 \%$ point after they are more than half grown, implying that water-bearing planetesimals are delivered preferentially late rather than early. Even more striking is the timing of accretion of embryos from beyond 2.5 AU. The large black dots give the planet mass following the impact of these embryos. Planet 4-2 is impacted by a body containing 2 embryos from beyond $2.5 \mathrm{AU}$, and only 3 planets do not experience the impact of an embryo from beyond 2.5 AU. In most cases, the impact of an embryo from beyond 2.5 AU is the final embryo impact on a planet. Planetesimals from beyond 2.5 AU that are 'pre-accreted' by another embryo before hitting the planet are not counted in this graph. Figure from O'Brien et al. (2006)

after the final giant impact on a given planet. For the CJS simulations, they find a median value of $1.2 \%$ for planets that are comparable in mass to the Earth, reasonably consistent with the Drake and Righter (2002) estimate. The median mass fraction of material from beyond 2.5 AU that arrives after the last giant impact, as a fraction of planet mass, is $0.7 \%$, and ranges from $0-2.9 \%$, whereas the total median mass fraction of material from beyond $2.5 \mathrm{AU}$ is $15 \%$. Thus, the vast majority of water-bearing material arrives prior to the late veneer in these simulations.

As can be seen in Fig. 4, the simulations of O'Brien et al. (2006) do not do a good job of reproducing Mars - even in the EJS simulations, the best Mars analogues are at least several times too massive. With larger numbers of EJS and CJS simulations (50 each), Fischer and Ciesla (2014) found that Mars-like planets could be produced in a small fraction of cases, but it is a very low-probability event, especially in the CJS case. This is an issue for nearly all simulations of the classical scenario, except in the case where Jupiter is made to be substantially more eccentric than its current value (Raymond et al. 2009). This issue was part of the motivation for the work of Hansen (2009) and the development of the Grand Tack model of Walsh et al. (2011) described in Sect. 3. O'Brien et al. (2014) extended the simulations of Walsh et al. (2011) to investigate water delivery in the Grand Tack scenario in more detail. Here we describe their key results.

The Walsh et al. (2011) simulations began with a population of embryos with $1 / 2$ or $1 / 4$ of a Mars mass and a swarm of planetesimals extending out to $3 \mathrm{AU}$, and populations of primitive planetesimals between the four giant planets (the belts) and beyond Neptune (the 
disk). The inward and outward migration of the giant planets pushes much of the inner Solar System material to inside of $1 \mathrm{AU}$, roughly reproducing the initial conditions of Hansen (2009), scatters material out of and then back into the asteroid belt, and scatters some of the material from the belt and disk populations into the terrestrial planet region as well. Walsh et al. (2011) did not explicitly track the accretion of the belt and disk material, but estimated that sufficient water would be delivered to explain the Earth's water budget. O'Brien et al. (2014) took the simulations of Walsh et al. (2011) immediately following the inward-thenoutward migration of the giant planets and integrated them to $150 \mathrm{Myr}$, explicitly including the material originating from between and beyond the giant planets. 16 planetary systems were generated.

O'Brien et al. (2014) find that for planets larger than $0.75 \mathrm{M}_{\oplus}$ the median fraction of material originating from the belts population $f_{\text {belts }}$ is $2.3 \%$, and for the disk population $f_{\text {disk }}$ is $0.7 \%$. Because the masses of the belt and disk populations were normalized such that each could individually explain the mass of C-type asteroids in the main belt, the total mass fraction of material from the giant planet region that ends up in the terrestrial planets is not the sum of $f_{\text {belts }}$ and $f_{\text {disk }}$, but rather a value between the two (i.e. between 0.7 and $2.3 \%$ ). Note that if the primitive planetesimals are similar to carbonaceous chondrites, these values are consistent with the estimates of Drake and Righter (2002) and Marty (2012) that carbonaceous chondrites only contributed about $2 \%$ of the Earth's total mass.

For the same assumption as made for the O'Brien et al. (2006) simulations, that the primitive bodies have a water mass fraction of $10 \%$ and no water is lost in collisions, Earth-mass planets would have a median water content of $2.3 \times 10^{-3} \mathrm{M}_{\oplus}$ if the water were delivered entirely by the belts population or $7 \times 10^{-4} \mathrm{M}_{\oplus}$ if it were delivered entirely by the disk population (and most likely a value somewhere between the two). Both values exceed the lower limit of $5 \times 10^{-4} \mathrm{M}_{\oplus}$ discussed in Sect. 2 (4.6 and 1.4 times larger, respectively). We note that the Grand Tack scenario has the important feature of matching both the water budget of Earth and the mass of C-type asteroids in the main belt in a self-consistent manner-a constraint that any model of water delivery by carbonaceous chondrite material must be able to match.

The $10 \%$ value assumed above for the water content of primitive planetesimals originating from the giant planet region may be conservative, given that main-belt comets may have a much larger water content than this (Jewitt 2012). The discovery of water vapor emanating from Ceres (Küppers et al. 2014) and the detection of water ice on the surface of Themis (Campins et al. 2010; Rivkin and Emery 2010) also suggest that the water content of primitive material may be larger than estimated from meteorites (in which only water bound to the silicates has survived). Thus, larger water contents are a possibility, and would not be inconsistent with the Earth's total water budget since several tens of oceans worth of water can potentially be stored in the mantle, as discussed in Sect. 2. A higher planetesimal water content could also allow for significant impact-related losses of water, while still delivering the minimum required amount.

Figure 6 shows the timing of accretion of water-bearing material. $f_{\text {belts }}$ and $f_{\text {disk }}$ are plotted vs. time for all planets whose final mass is larger than $0.75 \mathrm{M}_{\oplus}$. The time axis is in terms of normalized accretion time, which is the mass of the planet as a fraction of its final mass. If equal amounts of primitive material were accreted during each impact, all lines on the plot would be horizontal; the fact that the curves in Fig. 6 all increase with increasing time means that the primitive planetesimals preferentially arrive late in the planets' accretion. The delivery of primitive material late in the accretion process, when the planet is larger, means that water and volatiles that may be delivered by those impactors are more likely to be retained by the planet. Wood et al. (2008) and Rubie et al. (2011) show that material accreting to 

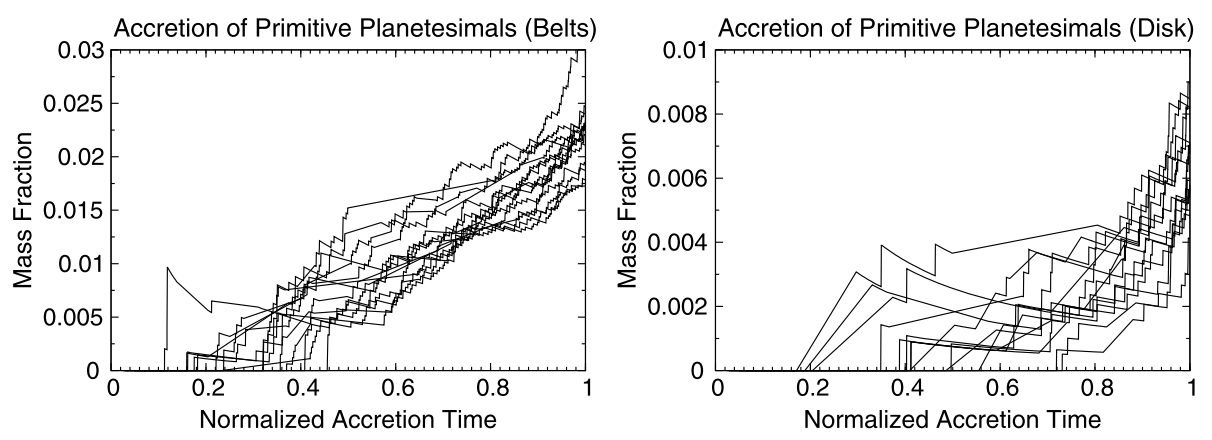

Fig. 6 Accretion curves for all planets larger than $0.75 \mathrm{M}_{\oplus}$ in the Grand Tack simulations of O’Brien et al. (2014). The mass fraction of primitive planetesimals from both the 'belts' and 'disk' populations are shown, where the belts population originally lies between the orbits of the giant planets, and the disk population initially lies beyond them. Accreted fractions are plotted vs. normalized time, which is the mass of the planet divided by its final mass. The curves all increase with time, which shows that the primitive material, and hence water and other volatiles, arrives relatively late during the accretion process. Figure from O'Brien et al. (2014)

form Earth was initially highly reduced, and the oxidation state increased with time, consistent with late addition of water to the terrestrial planets. Rubie et al. (2015b) integrated their multistage core-mantle differentiation model with actual Grand Tack simulations, and found that they can produce Earth-like planets with mantle concentrations of non-volatile elements and water that are close to the estimated values for the Earth's primitive mantle.

As with the CJS and EJS simulations described previously, the fact that primitive material is delivered late does not necessarily mean that water is all delivered as a late veneer at the tail end of accretion. Water delivery is still a relatively gradual process, with the planets continuing to grow while accreting water-rich planetesimals. The late veneer mass that is accreted in these simulations, however, tends to be significantly larger than in the CJS simulations, which were reasonably consistent with the estimate of $\lesssim 1 \%$ total mass from Drake and Righter (2002). Planets larger than $0.75 \mathrm{M}_{\oplus}$ have a median late veneer mass (defined as mass accreted after the last embryo impact) of $18 \%$, only a fraction of which is primitive water-bearing material. This high value is in part due the fact that many planets experience their last embryo impact in less than $20 \mathrm{Myr}$, and as shown by Jacobson et al. (2014), there tends to be an inverse correlation between late veneer mass and the time of last giant impact. Of the 27 planets larger than $0.5 \mathrm{M}_{\oplus}$ formed in the O'Brien et al. (2014) simulations, only 8 have last giant impacts after $20 \mathrm{Myr}$ and 5 of those have late veneer masses of a few percent or less. Jacobson et al. (2014) have shown that the timing of the last giant impact and the late veneer mass can be strongly affected by the initial embryo mass and embryo/planetesimal mass ratio. This suggests a wider range of initial conditions should be explored to determine which parameters are able to best fit all available constraints.

Raymond and Izidoro (2017a) recently proposed a new scenario for water delivery by planetesimals scattered inward during the giant planets' growth and migration, which has implications for the other models discussed above. Jupiter and Saturn experienced a phase of runaway gas accretion during which the planets' masses increased dramatically, until they carved a gap in the disk and accretion slowed (e.g. Lissauer et al. 2009). This growth was likely accompanied by orbital migration, although there are a number of viable migration histories (Pierens et al. 2014). The giant planets' growth invariably destabilizes the orbits of nearby planetesimals, gravitationally scattering them in all directions onto eccentric orbits. Given the dissipative effects of aerodynamic gas drag (Adachi et al. 1976), planetes- 
imals' eccentricities may be damped, decoupling the planetesimals from the giant planets and preventing further scattering. A fraction of planetesimals is naturally deposited interior to Jupiter's orbit, preferentially populating the outer part of the main asteroid belt (Raymond and Izidoro 2017a). Implanted planetesimals originate mainly between 4 and $10 \mathrm{AU}$ (but with a tail out to 15-20 AU) and match the orbits of the C-type asteroids. In fact, Ceres is located immediately adjacent to the peak in the distribution of $1000 \mathrm{~km}$-class planetesimals implanted by this mechanism (Raymond and Izidoro 2017a). Some planetesimals are scattered interior to the asteroid belt by Jupiter, crossing the orbits of the terrestrial planets. This preferentially happens for large planetesimals and later in the disk lifetime, when gas drag is weaker.

The scenario proposed in Raymond and Izidoro (2017a) would occur before any significant planetary growth in the terrestrial planet region, and hence could fit within any model of terrestrial planet formation. In the Grand Tack model, for example, there would be an additional, earlier scattering of water-rich material into the terrestrial planet region by the Raymond and Izidoro (2017a) mechanism, in addition to the later scattering of material during the inward and outward migration of Jupiter. The amount of material scattered inwards during this early phase, however, is unconstrained since it is based on the (unknown) planetesimal distribution in the primordial Jupiter-Saturn zone. Likewise, the timing of accretion of that material is unclear - even though it is implanted early, it may be accreted relatively late, especially if it is on eccentric orbits. Future modeling of terrestrial planet formation incorporating the Raymond and Izidoro (2017a) scenario, along with geochemical constraints (e.g. Rubie et al. 2015b), may help better understand the magnitude and effects of this early planetesimal scattering process.

\section{Summary and Conclusions}

We have reviewed the main scenarios for delivery of water to the terrestrial planets. Several early/in-situ mechanisms have been proposed, including the incorporation of water into olivine grains around $1 \mathrm{AU}$ through adsorption directly from the gaseous nebula (Stimpfl et al. 2006; Muralidharan et al. 2008; King et al. 2010; Asaduzzaman et al. 2015), oxidation of an early hydrogen atmosphere by $\mathrm{FeO}$ in the terrestrial magma ocean (Ikoma and Genda 2006; Genda and Ikoma 2008), and incorporation of inward-drifting phyllosilicate dust into planetesimals around $1 \mathrm{AU}$ (Ciesla and Lauretta 2005). As discussed in Sect. 4, each of these scenarios has its problems, for example potential inconsistencies with the measured $\mathrm{D} / \mathrm{H}$ ratio of the Earth, and all run counter to work showing that the material that first accreted to form the Earth was highly reduced (Wood et al. 2008; Rubie et al. 2011). Cometary delivery has also been proposed, as discussed in Sect. 5. It too may have issues matching the measured $\mathrm{D} / \mathrm{H}$ of the Earth, but a bigger issue is that the delivery efficiency of comets is very low (Morbidelli et al. 2000), such that even delivering a single Earth ocean of water is unlikely.

Later-stage delivery of water-bearing material, in the course of planetary accretion, is a more promising scenario (Sect. 6). In the classical scenario, with planetesimals and embryos extending through the outer asteroid belt region, substantial amounts of water can be delivered to the terrestrial planets if Jupiter and Saturn are on circular, low-eccentricity orbits as predicted by the Nice Model (e.g. O'Brien et al. 2006; Raymond et al. 2009). However, it is possible that so much carbonaceous-chondrite-like material could be delivered in that case that the volatile element abundances and oxygen isotope ratios of the final planets are not consistent with Earth's values (Drake and Righter 2002; Marty 2012). Such simulations 
are also not able to reproduce the small mass of Mars. In the Grand Tack scenario (Walsh et al. 2011; O'Brien et al. 2014), where inward and outward migration of the giant planets truncates the terrestrial planetesimal/embryo distribution and scatters material inwards from the giant planet region, the final terrestrial planet systems are a better match to the actual Solar System (with a small Mars). Sufficient water delivery occurs to explain the Earth's water budget without delivering excessive carbonaceous material that would violate the constraints of Drake and Righter (2002) and Marty (2012) regarding volatile elements and oxygen isotope ratios. Water is delivered relatively late in the accretion process, but not as a late veneer. The later addition of water is consistent with evidence that the earliest material accreted to the Earth was highly reduced, and later material was more oxidized (Wood et al. 2008; Rubie et al. 2011, 2015b). While some issues still remain with the Grand Tack scenario, specifically regarding the accretion timescales of the planets that form, this discrepancy may be reduced as a wider range of initial conditions is explored (e.g. Jacobson et al. 2014). Finally, new models of terrestrial planet formation have been proposed since the introduction of the Grand Tack scenario, such as those based on pebble accretion (e.g. Levison et al. 2015) or having an initially low mass in the asteroid belt region (Izidoro et al. 2014, 2015; Dra̧żkowska et al. 2016; Morbidelli and Raymond 2016; Raymond and Izidoro 2017b), as well as new mechanisms for water delivery, such as early inward scattering of planetesimals during the formation of the giant planets (Raymond and Izidoro 2017a). These may be promising avenues for future research.

Acknowledgements We thank the International Space Science Institute (ISSI) for organizing and supporting the workshop "The Delivery of Water to Protoplanets, Planets and Satellites", and an anonymous reviewer for their helpful comments and suggestions. AI acknowledges financial support from FAPESP through grants number 16/12686-2 and 16/19556-7. SNR acknowledges Agence Nationale pour la Recherche grant ANR-13-BS05-0003-002 (grant MOJO). DCR and SAJ were supported by the European Research Council Advanced Grant "ACCRETE" (contract number 290568), and additional support to DCR was provided by the German Science Foundation (DFG) Priority Programme SPP1833 "Building a Habitable Earth" (Ru1323/10-1).

\section{References}

Y. Abe, E. Ohtani, T. Okuchi, K. Righter, M. Drake, Water in the early earth, in Origin of the Earth and Moon, ed. by R.M. Canup, K. Righter (University of Arizona Press, Tucson, 2000), pp. 413-433

I. Adachi, C. Hayashi, K. Nakazawa, The gas drag effect on the elliptical motion of a solid body in the primordial solar nebula. Prog. Theor. Phys. 56, 1756-1771 (1976). https://doi.org/10.1143/PTP.56.1756

C.M.O.'D. Alexander, R. Bowden, M.L. Fogel, K.T. Howard, C.D.K. Herd, L.R. Nittler, The provenances of asteroids, and their contributions to the volatile inventories of the terrestrial planets. Science 337, 721 (2012). https://doi.org/10.1126/science.1223474

C.M.O.'D. Alexander, K.D. McKeegan, K. Altwegg, Water reservoirs in small planetary bodies: meteorites, asteroids, and comets. Space Sci. Rev. (2018, in press). https://doi.org/10.1007/s11214-018-0474-9

K. Altwegg, H. Balsiger, A. Bar-Nun, J.J. Berthelier, A. Bieler, P. Bochsler, C. Briois, U. Calmonte, M. Combi, J. De Keyser, P. Eberhardt, B. Fiethe, S. Fuselier, S. Gasc, T.I. Gombosi, K.C. Hansen, M. Hässig, A. Jäckel, E. Kopp, A. Korth, L. LeRoy, U. Mall, B. Marty, O. Mousis, E. Neefs, T. Owen, H. Rème, M. Rubin, T. Sémon, C.Y. Tzou, H. Waite, P. Wurz, 67P/Churyumov-Gerasimenko, a Jupiter family comet with a high D/H ratio. Science 347(27), 1261952 (2015). https://doi.org/10.1126/science. 1261952

Y. Amelin, A.N. Krot, I.D. Hutcheon, A.A. Ulyanov, Lead isotopic ages of chondrules and calciumaluminum-rich inclusions. Science 297, 1678-1683 (2002). https://doi.org/10.1126/science.1073950

P.J. Armitage, Dynamics of protoplanetary disks. Annu. Rev. Astron. Astrophys. 49, 195-236 (2011). https:// doi.org/10.1146/annurev-astro-081710-102521

A. Asaduzzaman, K. Muralidharan, J. Ganguly, Incorporation of water into olivine during nebular condensation: insights from density functional theory and thermodynamics, and implications for phyllosilicate formation and terrestrial water inventory. Meteorit. Planet. Sci. 50, 578-589 (2015). https://doi.org/10. 1111/maps. 12409 
G. Avice, B. Marty, The iodine-plutonium-xenon age of the Moon-Earth system revisited. Philos. Trans. R. Soc. Lond. Ser. A 372, 20130260 (2014). https://doi.org/10.1098/rsta.2013.0260

J. Badro, A.S. Côté, J.P. Brodholt, A seismologically consistent compositional model of Earth's core. Proc. Natl. Acad. Sci. 111, 7542-7545 (2014). https://doi.org/10.1073/pnas.1316708111

K. Baillié, S. Charnoz, E. Pantin, Time evolution of snow regions and planet traps in an evolving protoplanetary disk. Astron. Astrophys. 577, 65 (2015). https://doi.org/10.1051/0004-6361/201424987

V.R. Baker, Geomorphological evidence for water on Mars. Elements 2(3), 139-143 (2007). https://doi.org/ 10.2113/gselements.2.3.139

B. Bitsch, A. Johansen, M. Lambrechts, A. Morbidelli, The structure of protoplanetary discs around evolving young stars. Astron. Astrophys. 575, 28 (2015). https://doi.org/10.1051/0004-6361/201424964

W.F. Bottke, D. Vokrouhlický, D. Minton, D. Nesvorný, A. Morbidelli, R. Brasser, B. Simonson, H.F. Levison, An Archaean heavy bombardment from a destabilized extension of the asteroid belt. Nature $\mathbf{4 8 5}$, 78-81 (2012). https://doi.org/10.1038/nature10967

A. Bouvier, M. Wadhwa, The age of the Solar System redefined by the oldest $\mathrm{Pb}-\mathrm{Pb}$ age of a meteoritic inclusion. Nat. Geosci. 3, 637-641 (2010). https://doi.org/10.1038/ngeo941

R. Brasser, S. Matsumura, S. Ida, S.J. Mojzsis, S.C. Werner, Analysis of terrestrial planet formation by the Grand Tack Model: system architecture and tack location. Astrophys. J. 821, 75 (2016). https://doi.org/ 10.3847/0004-637X/821/2/75

B.C. Bromley, S.J. Kenyon, A hybrid N-body-coagulation code for planet formation. Astron. J. 131, 27372748 (2006). https://doi.org/10.1086/503280

T.H. Burbine, T.J. McCoy, A. Meibom, B. Gladman, K. Keil, Meteoritic parent bodies: their number and identification, in Asteroids III, ed. by W.F. Bottke Jr., A. Cellino, P. Paolicchi, R.P. Binzel (University of Arizona Press, Tucson, 2002), pp. 653-667

A.G.W. Cameron, Higher-resolution Simulations of the Giant Impact, in Origin of the Earth and Moon, ed. by R.M. Canup, K. Righter, et al.(University of Arizona Press, Tucson, 2000), pp. 133-144

H. Campins, K. Hargrove, N. Pinilla-Alonso, E.S. Howell, M.S. Kelley, J. Licandro, T. Mothé-Diniz, Y. Fernández, J. Ziffer, Water ice and organics on the surface of the asteroid 24 Themis. Nature 464, 13201321 (2010). https://doi.org/10.1038/nature09029

R.M. Canup, Simulations of a late lunar-forming impact. Icarus 168, 433-456 (2004). https://doi.org/10.1016/ j.icarus.2003.09.028

R.M. Canup, Forming a Moon with an Earth-like composition via a giant impact. Science 338, 1052-1055 (2012). https://doi.org/10.1126/science.1226073

R.M. Canup, E. Asphaug, Origin of the Moon in a giant impact near the end of the Earth's formation. Nature 412, 708-712 (2001). https://doi.org/10.1038/35089010

J.E. Chambers, Making more terrestrial planets. Icarus 152, 205-224 (2001). https://doi.org/10.1006/icar. 2001.6639

J.E. Chambers, Planetary accretion in the inner Solar System. Earth Planet. Sci. Lett. 223, 241-252 (2004). https://doi.org/10.1016/j.eps1.2004.04.031

J.E. Chambers, Planetesimal formation by turbulent concentration. Icarus 208, 505-517 (2010). https://doi. org/10.1016/j.icarus.2010.03.004

J.E. Chambers, Pebble accretion and the diversity of planetary systems. Astrophys. J. 825, 63 (2016). https:// doi.org/10.3847/0004-637X/825/1/63

J.E. Chambers, D.P. O'Brien, A.M. Davis, Accretion of planetesimals and the formation of rocky planets, in Protoplanetary Dust: Astrophysical and Cosmochemical Perspectives, ed. by D.A. Apai, D.S. Lauretta (2010), pp. 299-335

C.R. Chapman, B.A. Cohen, D.H. Grinspoon, What are the real constraints on the existence and magnitude of the late heavy bombardment? Icarus 189, 233-245 (2007). https://doi.org/10.1016/j.icarus.2006.12.020

C.F. Chyba, The cometary contribution to the oceans of primitive earth. Nature 330, 632-635 (1987). https:// doi.org/10.1038/330632a0

F. Ciesla, D. Lauretta, Radial migration and dehydration of phyllosilicates in the solar nebula. Earth Planet. Sci. Lett. 231, 1-8 (2005). https://doi.org/10.1016/j.epsl.2004.12.022

J.N. Connelly, M. Bizzarro, A.N. Krot, Å. Nordlund, D. Wielandt, M.A. Ivanova, The absolute chronology and thermal processing of solids in the solar protoplanetary disk. Science 338, 651-655 (2012). https:// doi.org/10.1126/science. 1226919

M. Ćuk, S.T. Stewart, Making the Moon from a fast-spinning Earth: a giant impact followed by resonant despinning. Science 338, 1047-1052 (2012). https://doi.org/10.1126/science.1225542

J.N. Cuzzi, R.C. Hogan, J.M. Paque, A.R. Dobrovolskis, Size-selective concentration of chondrules and other small particles in protoplanetary nebula turbulence. Astrophys. J. 546, 496-508 (2001). https://doi.org/ $10.1086 / 318233$

J.N. Cuzzi, R.C. Hogan, K. Shariff, Toward planetesimals: dense chondrule clumps in the protoplanetary nebula. Astrophys. J. 687, 1432-1447 (2008). https://doi.org/10.1086/591239 
G. D'Angelo, F. Marzari, Outward migration of Jupiter and Saturn in evolved gaseous disks. Astrophys. J. 757, 50 (2012). https://doi.org/10.1088/0004-637X/757/1/50

N. Dauphas, A. Pourmand, Hf-W-Th evidence for rapid growth of Mars and its status as a planetary embryo. Nature 473, 489-492 (2011). https://doi.org/10.1038/nature10077

N. Dauphas, F. Robert, B. Marty, The late asteroidal and cometary bombardment of earth as recorded in water deuterium to protium ratio. Icarus 148, 508-512 (2000). https://doi.org/10.1006/icar.2000.6489

S.S. Davis, Condensation front migration in a protoplanetary nebula. Astrophys. J. 620, 994-1001 (2005). https://doi.org/10.1086/427073

M.C. de Sanctis, A. Raponi, E. Ammannito, M. Ciarniello, M.J. Toplis, H.Y. McSween, J.C. Castillo-Rogez, B.L. Ehlmann, F.G. Carrozzo, S. Marchi, F. Tosi, F. Zambon, F. Capaccioni, M.T. Capria, S. Fonte, M. Formisano, A. Frigeri, M. Giardino, A. Longobardo, G. Magni, E. Palomba, L.A. McFadden, C.M. Pieters, R. Jaumann, P. Schenk, R. Mugnuolo, C.A. Raymond, C.T. Russell, Bright carbonate deposits as evidence of aqueous alteration on (1) Ceres. Nature 536, 54-57 (2016). https://doi.org/10.1038/ nature 18290

R. Deienno, R.S. Gomes, K.J. Walsh, A. Morbidelli, D. Nesvorný, Is the Grand Tack model compatible with the orbital distribution of main belt asteroids? Icarus 272, 114-124 (2016). https://doi.org/10.1016/ j.icarus.2016.02.043

A.H. Delsemme, Cometary origin of carbon and water on the terrestrial planets. Adv. Space Res. 12, 5-12 (1992). https://doi.org/10.1016/0273-1177(92)90147-P

A. Delsemme, The origin of the atmosphere and of the oceans, in Comets and the Origin and Evolution of Life, ed. by P.J. Thomas, C.F. Chyba, C.P. McKay (Springer, New York, 1997), pp. 29-67

A.H. Delsemme, The deuterium enrichment observed in recent comets is consistent with the cometary origin of seawater. Planet. Space Sci. 47, 125-131 (1998). https://doi.org/10.1016/S0032-0633(98)00093-2

T.M. Donahue, J.H. Hoffman, R.R. Hodges, A.J. Watson, Venus was wet-a measurement of the ratio of deuterium to hydrogen. Science 216, 630-633 (1982). https://doi.org/10.1126/science.216.4546.630

M.J. Drake, K. Righter, Determining the composition of the Earth. Nature 416, 39-44 (2002). https://doi.org/ $10.1038 / 416039$ a

J. Drążkowska, Y. Alibert, B. Moore, Close-in planetesimal formation by pile-up of drifting pebbles. Astron. Astrophys. 594, 105 (2016). https://doi.org/10.1051/0004-6361/201628983

G. Dreibus, H. Waenke, Supply and loss of volatile constituents during the accretion of terrestrial planets, in Origin and Evolution of Planetary and Satellite Atmospheres, ed. by S.K. Atreya, J.B. Pollack, M.S. Matthews (University of Arizona Press, Tucson, 1989), pp. 268-288

W.C. Feldman, T.H. Prettyman, S. Maurice, J.J. Plaut, D.L. Bish, D.T. Vaniman, M.T. Mellon, A.E. Metzger, S.W. Squyres, S. Karunatillake, W.V. Boynton, R.C. Elphic, H.O. Funsten, D.J. Lawrence, R.L. Tokar, Global distribution of near-surface hydrogen on Mars. J. Geophys. Res., Planets 109, 09006 (2004). https://doi.org/10.1029/2003JE002160

R.A. Fischer, F.J. Ciesla, Dynamics of the terrestrial planets from a large number of N-body simulations. Earth Planet. Sci. Lett. 392, 28-38 (2014). https://doi.org/10.1016/j.epsl.2014.02.011

J. Ganguly, A. Asaduzzaman, K. Muralidharan, Origin of water in Earth with high D/H ratio relative to protosolar nebula, and an explanation of its similarity with the isotopic ratios of carbonaceous chondrites and asteroid Vesta, in 79th Annual Meeting of the Meteoritical Society. LPI Contributions, vol. 1921 (2016), p. 6055

P. Garaud, D.N.C. Lin, The effect of internal dissipation and surface irradiation on the structure of disks and the location of the snow line around Sun-like stars. Astrophys. J. 654, 606-624 (2007). https://doi.org/ $10.1086 / 509041$

H. Genda, M. Ikoma, Origin of the ocean on the Earth: early evolution of water D/H in a hydrogen-rich atmosphere. Icarus 194, 42-52 (2008). https://doi.org/10.1016/j.icarus.2007.09.007

P. Goldreich, S. Tremaine, Disk-satellite interactions. Astrophys. J. 241, 425-441 (1980). https://doi.org/ $10.1086 / 158356$

P. Goldreich, W.R. Ward, The formation of planetesimals. Astrophys. J. 183, 1051-1062 (1973). https://doi. org/10.1086/152291

R.S. Gomes, A. Morbidelli, H.F. Levison, Planetary migration in a planetesimal disk: why did Neptune stop at 30 AU? Icarus 170, 492-507 (2004). https://doi.org/10.1016/j.icarus.2004.03.011

R. Gomes, H.F. Levison, K. Tsiganis, A. Morbidelli, Origin of the cataclysmic Late Heavy Bombardment period of the terrestrial planets. Nature 435, 466-469 (2005). https://doi.org/10.1038/nature03676

J. Gradie, E. Tedesco, Compositional structure of the asteroid belt. Science 216, 1405-1407 (1982). https://doi.org/10.1126/science.216.4553.1405

R. Greenberg, W.K. Hartmann, C.R. Chapman, J.F. Wacker, Planetesimals to planets-numerical simulation of collisional evolution. Icarus 35, 1-26 (1978). https://doi.org/10.1016/0019-1035(78)90057-X

J.M. Hahn, R. Malhotra, Orbital evolution of planets embedded in a planetesimal disk. Astron. J. 117, 30413053 (1999). https://doi.org/10.1086/300891 
K.E. Haisch Jr., E.A. Lada, C.J. Lada, Disk frequencies and lifetimes in young clusters. Astrophys. J. Lett. 553, 153-156 (2001). https://doi.org/10.1086/320685

L.J. Hallis, G.R. Huss, K. Nagashima, G.J. Taylor, S.A. Halldórsson, D.R. Hilton, M.J. Mottl, K.J. Meech, Evidence for primordial water in Earth's deep mantle. Science 350, 795-797 (2015). https://doi.org/ $10.1126 /$ science.aac4834

B.M.S. Hansen, Formation of the terrestrial planets from a narrow annulus. Astrophys. J. 703, 1131-1140 (2009). https://doi.org/10.1088/0004-637X/703/1/1131

P. Hartogh, D.C. Lis, D. Bockelée-Morvan, M. de Val-Borro, N. Biver, M. Küppers, M. Emprechtinger, E.A. Bergin, J. Crovisier, M. Rengel, R. Moreno, S. Szutowicz, G.A. Blake, Ocean-like water in the Jupiterfamily comet 103P/Hartley 2. Nature 478, 218-220 (2011). https://doi.org/10.1038/nature10519

R. Hueso, T. Guillot, Evolution of protoplanetary disks: constraints from DM Tauri and GM Aurigae. Astron. Astrophys. 442, 703-725 (2005). https://doi.org/10.1051/0004-6361:20041905

M. Ikoma, H. Genda, Constraints on the mass of a habitable planet with water of nebular origin. Astrophys. J. 648, 696-706 (2006). https://doi.org/10.1086/505780

A. Izidoro, K. de Souza Torres, O.C. Winter, N. Haghighipour, A compound model for the origin of Earth's water. Astrophys. J. 767, 54 (2013). https://doi.org/10.1088/0004-637X/767/1/54

A. Izidoro, N. Haghighipour, O.C. Winter, M. Tsuchida, Terrestrial planet formation in a protoplanetary disk with a local mass depletion: a successful scenario for the formation of Mars. Astrophys. J. 782, 31 (2014). https://doi.org/10.1088/0004-637X/782/1/31

A. Izidoro, S.N. Raymond, A. Morbidelli, O.C. Winter, Terrestrial planet formation constrained by Mars and the structure of the asteroid belt. Mon. Not. R. Astron. Soc. 453, 3619-3634 (2015). https://doi.org/10. 1093/mnras/stv1835

A. Izidoro, S.N. Raymond, A. Pierens, A. Morbidelli, O.C. Winter, D. Nesvorny“, The asteroid belt as a relic from a chaotic early Solar System. Astrophys. J. 833, 40 (2016). https://doi.org/10.3847/1538$4357 / 833 / 1 / 40$

S.A. Jacobson, K.J. Walsh, Earth and terrestrial planet formation. American Geophysical Union Geophysical Monograph Series 212, 49-70 (2015). https://doi.org/10.1002/9781118860359.ch3

S.A. Jacobson, A. Morbidelli, S.N. Raymond, D.P. O'Brien, K.J. Walsh, D.C. Rubie, Highly siderophile elements in Earth's mantle as a clock for the Moon-forming impact. Nature 508, 84-87 (2014). https:// doi.org/10.1038/nature13172

D. Jewitt, The active asteroids. Astron. J. 143, 66 (2012). https://doi.org/10.1088/0004-6256/143/3/66

A. Johansen, J.S. Oishi, M. Mac Low, H. Klahr, T. Henning, A. Youdin, Rapid planetesimal formation in turbulent circumstellar disks. Nature 448, 1022-1025 (2007). https://doi.org/10.1038/nature06086

H.E. King, M. Stimpfl, P. Deymier, M.J. Drake, C.R.A. Catlow, A. Putnis, N.H. de Leeuw, Computer simulations of water interactions with low-coordinated forsterite surface sites: implications for the origin of water in the inner solar system. Earth Planet. Sci. Lett. 300, 11-18 (2010). https://doi.org/10. 1016/j.eps1.2010.10.019

N.T. Kita, G.R. Huss, S. Tachibana, Y. Amelin, L.E. Nyquist, I.D. Hutcheon, Constraints on the origin of chondrules and CAIs from short-lived and long-lived radionuclides, in Chondrites and the Protoplanetary Disk, ed. by A.N. Krot, E.R.D. Scott, B. Reipurth. Astronomical Society of the Pacific Conference Series, vol. 341 (2005), pp. 558-587

T. Kleine, M. Touboul, B. Bourdon, F. Nimmo, K. Mezger, H. Palme, S.B. Jacobsen, Q.Z. Yin, A.N. Halliday, Hf-W chronology of the accretion and early evolution of asteroids and terrestrial planets. Geochim. Cosmochim. Acta 73, 5150-5188 (2009). https://doi.org/10.1016/j.gca.2008.11.047

W. Kley, R.P. Nelson, Planet-disk interaction and orbital evolution. Annu. Rev. Astron. Astrophys. 50, 211249 (2012). https://doi.org/10.1146/annurev-astro-081811-125523

E. Kokubo, S. Ida, On runaway growth of planetesimals. Icarus 123, 180-191 (1996). https://doi.org/10.1006/ icar. 1996.0148

E. Kokubo, S. Ida, Oligarchic growth of protoplanets. Icarus 131, 171-178 (1998). https://doi.org/10.1006/ icar. 1997.5840

E. Kokubo, S. Ida, Formation of protoplanets from planetesimals in the solar nebula. Icarus 143, 15-27 (2000). https://doi.org/10.1006/icar.1999.6237

J. Kominami, S. Ida, The effect of tidal interaction with a gas disk on formation of terrestrial planets. Icarus 157, 43-56 (2002). https://doi.org/10.1006/icar.2001.6811

J. Kominami, S. Ida, Formation of terrestrial planets in a dissipating gas disk with Jupiter and Saturn. Icarus 167, 231-243 (2004). https://doi.org/10.1016/j.icarus.2003.10.005

M. Küppers, L. O’Rourke, D. Bockelée-Morvan, V. Zakharov, S. Lee, P. von Allmen, B. Carry, D. Teyssier, A. Marston, T. Müller, J. Crovisier, M.A. Barucci, R. Moreno, Localized sources of water vapour on the dwarf planet (1) Ceres. Nature 505, 525-527 (2014). https://doi.org/10.1038/nature12918

M. Lambrechts, A. Johansen, Rapid growth of gas-giant cores by pebble accretion. Astron. Astrophys. 544, 32 (2012). https://doi.org/10.1051/0004-6361/201219127 
M. Lambrechts, A. Johansen, Forming the cores of giant planets from the radial pebble flux in protoplanetary discs. Astron. Astrophys. 572, 107 (2014). https://doi.org/10.1051/0004-6361/201424343

C. Lécuyer, The hydrogen isotope composition of seawater and the global water cycle. Chem. Geol. 145, 249-261 (1998). https://doi.org/10.1016/S0009-2541(97)00146-0

H.F. Levison, K.A. Kretke, K.J. Walsh, W.F. Bottke, Growing the terrestrial planets from the gradual accumulation of sub-meter sized objects. Proc. Natl. Acad. Sci. 112, 14180-14185 (2015). https://doi.org/ $10.1073 /$ pnas. 1513364112

D.N.C. Lin, J. Papaloizou, On the tidal interaction between protoplanets and the protoplanetary disk. IIIOrbital migration of protoplanets. Astrophys. J. 309, 846-857 (1986). https://doi.org/10.1086/164653

D.C. Lis, N. Biver, D. Bockelée-Morvan, P. Hartogh, E.A. Bergin, G.A. Blake, J. Crovisier, M. de Val-Borro, E. Jehin, M. Küppers, J. Manfroid, R. Moreno, M. Rengel, S. Szutowicz, A Herschel study of D/H in water in the Jupiter-family comet 45P/Honda-Mrkos-Pajdušáková and Prospects for D/H Measurements with CCAT. Astrophys. J. Lett. 774, 3 (2013). https://doi.org/10.1088/2041-8205/774/1/L3

J.J. Lissauer, O. Hubickyj, G. D'Angelo, P. Bodenheimer, Models of Jupiter's growth incorporating thermal and hydrodynamic constraints. Icarus 199, 338-350 (2009). https://doi.org/10.1016/j.icarus. 2008.10.004

P.S. Lykawka, T. Ito, Terrestrial planet formation during the migration and resonance crossings of the giant planets. Astrophys. J. 773, 65 (2013). https://doi.org/10.1088/0004-637X/773/1/65

R. Malhotra, The origin of Pluto's peculiar orbit. Nature 365, 819-821 (1993). https://doi.org/10.1038/ 365819a0

R. Malhotra, The origin of Pluto's orbit: implications for the solar system beyond Neptune. Astron. J. 110, 420-429 (1995). https://doi.org/10.1086/117532

S. Marchi, W.F. Bottke, D.A. Kring, A. Morbidelli, The onset of the lunar cataclysm as recorded in its ancient crater populations. Earth Planet. Sci. Lett. 325, 27-38 (2012). https://doi.org/10.1016/j.epsl. 2012.01.021

B. Marty, The origins and concentrations of water, carbon, nitrogen and noble gases on Earth. Earth Planet. Sci. Lett. 313, 56-66 (2012). https://doi.org/10.1016/j.eps1.2011.10.040

F. Masset, M. Snellgrove, Reversing type II migration: resonance trapping of a lighter giant protoplanet. Mon. Not. R. Astron. Soc. 320, 55-59 (2001). https://doi.org/10.1046/j.1365-8711.2001.04159.x

T.B. McCord, C. Sotin, Ceres: Evolution and current state. J. Geophys. Res., Planets 110, 5009 (2005). https:// doi.org/10.1029/2004JE002244

A. Morbidelli, A. Crida, The dynamics of Jupiter and Saturn in the gaseous protoplanetary disk. Icarus 191, 158-171 (2007). https://doi.org/10.1016/j.icarus.2007.04.001

A. Morbidelli, D. Nesvorny, Dynamics of pebbles in the vicinity of a growing planetary embryo: hydro-dynamical simulations. Astron. Astrophys. 546, 18 (2012). https://doi.org/10.1051/0004-6361/ 201219824

A. Morbidelli, S.N. Raymond, Challenges in planet formation. J. Geophys. Res., Planets 121, 1962-1980 (2016). https://doi.org/10.1002/2016JE005088

A. Morbidelli, J. Chambers, J.I. Lunine, J.M. Petit, F. Robert, G.B. Valsecchi, K.E. Cyr, Source regions and time scales for the delivery of water to Earth. Meteorit. Planet. Sci. 35, 1309-1320 (2000). https://doi.org/10.1111/j.1945-5100.2000.tb01518.x

A. Morbidelli, H.F. Levison, K. Tsiganis, R. Gomes, Chaotic capture of Jupiter's Trojan asteroids in the early Solar System. Nature 435, 462-465 (2005). https://doi.org/10.1038/nature03540

A. Morbidelli, R. Brasser, R. Gomes, H.F. Levison, K. Tsiganis, Evidence from the asteroid belt for a violent past evolution of Jupiter's orbit. Astron. J. 140, 1391-1401 (2010). https://doi.org/10.1088/ 0004-6256/140/5/1391

A. Morbidelli, S. Marchi, W.F. Bottke, D.A. Kring, A sawtooth-like timeline for the first billion years of lunar bombardment. Earth Planet. Sci. Lett. 355, 144-151 (2012a). https://doi.org/10.1016/j.eps1.2012.07.037

A. Morbidelli, J.I. Lunine, D.P. O’Brien, S.N. Raymond, K.J. Walsh, Building terrestrial planets. Annu. Rev. Earth Planet. Sci. 40, 251-275 (2012b). https://doi.org/10.1146/annurev-earth-042711-105319

A. Morbidelli, M. Lambrechts, S. Jacobson, B. Bitsch, The great dichotomy of the Solar System: Small terrestrial embryos and massive giant planet cores. Icarus 258, 418-429 (2015). https://doi.org/10.1016/ j.icarus.2015.06.003

A. Morbidelli, B. Bitsch, A. Crida, M. Gounelle, T. Guillot, S. Jacobson, A. Johansen, M. Lambrechts, E. Lega, Fossilized condensation lines in the Solar System protoplanetary disk. Icarus 267, 368-376 (2016). https://doi.org/10.1016/j.icarus.2015.11.027

R. Morishima, M.W. Schmidt, J. Stadel, B. Moore, Formation and accretion history of terrestrial planets from runaway growth through to late time: implications for orbital eccentricity. Astrophys. J. 685, 1247-1261 (2008). https://doi.org/10.1086/590948 
R. Morishima, J. Stadel, B. Moore, From planetesimals to terrestrial planets: N-body simulations including the effects of nebular gas and giant planets. Icarus 207, 517-535 (2010). https://doi.org/10.1016/ j.icarus.2009.11.038

T. Mothé-Diniz, J.M.Á. Carvano, D. Lazzaro, Distribution of taxonomic classes in the main belt of asteroids. Icarus 162, 10-21 (2003). https://doi.org/10.1016/S0019-1035(02)00066-0

K. Muralidharan, P. Deymier, M. Stimpfl, N.H. de Leeuw, M.J. Drake, Origin of water in the inner Solar System: a kinetic Monte Carlo study of water adsorption on forsterite. Icarus 198, 400-407 (2008). https:// doi.org/10.1016/j.icarus.2008.07.017

M. Nagasawa, D.N.C. Lin, E. Thommes, Dynamical shake-up of planetary systems. I. Embryo trapping and induced collisions by the sweeping secular resonance and embryo-disk tidal interaction. Astrophys. J. 635, 578-598 (2005). https://doi.org/10.1086/497386

R. Nomura, K. Hirose, K. Uesugi, Y. Ohishi, A. Tsuchiyama, A. Miyake, Y. Ueno, Low core-mantle boundary temperature inferred from the solidus of pyrolite. Science 343(6170), 522-525 (2014). https://doi.org/ $10.1126 /$ science. 1248186

D.P. O'Brien, M.V. Sykes, The origin and evolution of the asteroid belt-implications for Vesta and Ceres. Space Sci. Rev. 163, 41-61 (2011). https://doi.org/10.1007/s11214-011-9808-6

D.P. O'Brien, A. Morbidelli, H.F. Levison, Terrestrial planet formation with strong dynamical friction. Icarus 184, 39-58 (2006). https://doi.org/10.1016/j.icarus.2006.04.005

D.P. O'Brien, A. Morbidelli, W.F. Bottke, The primordial excitation and clearing of the asteroid beltrevisited. Icarus 191, 434-452 (2007). https://doi.org/10.1016/j.icarus.2007.05.005

D.P. O'Brien, K.J. Walsh, A. Morbidelli, S.N. Raymond, A.M. Mandell, Water delivery and giant impacts in the 'Grand Tack' scenario. Icarus 239, 74-84 (2014). https://doi.org/10.1016/j.icarus.2014.05.009

M. Ogihara, S. Ida, A. Morbidelli, Accretion of terrestrial planets from oligarchs in a turbulent disk. Icarus 188, 522-534 (2007). https://doi.org/10.1016/j.icarus.2006.12.006

A. Oka, T. Nakamoto, S. Ida, Evolution of snow line in optically thick protoplanetary disks: effects of water ice opacity and dust grain size. Astrophys. J. 738, 141 (2011). https://doi.org/10.1088/0004-637X/ 738/2/141

C.W. Ormel, H.H. Klahr, The effect of gas drag on the growth of protoplanets. Analytical expressions for the accretion of small bodies in laminar disks. Astron. Astrophys. 520, 43 (2010). https://doi.org/10.1051/ 0004-6361/201014903

T. Owen, A. Bar-Nun, Comets, impacts and atmospheres. Icarus 116, 215-226 (1995). https://doi.org/10. 1006/icar.1995.1122

A.H. Peslier, M. Schönbächler, H. Busemann, S.I. Karato, Water in the Earth's interior: distribution and origin. Space Sci. Rev. 212, 743-810 (2017). https://doi.org/10.1007/s11214-017-0387-Z

J. Petit, A. Morbidelli, J. Chambers, The primordial excitation and clearing of the asteroid belt. Icarus 153, 338-347 (2001). https://doi.org/10.1006/icar.2001.6702

A. Pierens, R.P. Nelson, Constraints on resonant-trapping for two planets embedded in a protoplanetary disc. Astron. Astrophys. 482, 333-340 (2008). https://doi.org/10.1051/0004-6361:20079062

A. Pierens, S.N. Raymond, Two phase, inward-then-outward migration of Jupiter and Saturn in the gaseous solar nebula. Astron. Astrophys. 533, 131 (2011). https://doi.org/10.1051/0004-6361/201117451

A. Pierens, S.N. Raymond, D. Nesvorny, A. Morbidelli, Outward migration of Jupiter and Saturn in 3:2 or 2:1 resonance in radiative disks: implications for the Grand Tack and Nice models. Astrophys. J. Lett. 795, 11 (2014). https://doi.org/10.1088/2041-8205/795/1/L11

S.N. Raymond, A. Izidoro, Origin of water in the inner Solar System: planetesimals scattered inward during Jupiter and Saturn's rapid gas accretion. Icarus 297, 134-148 (2017a). https://doi.org/10.1016/ j.icarus.2017.06.030

S.N. Raymond, A. Izidoro, The empty primordial asteroid belt. Sci. Adv. 3, 1701138 (2017b). https://doi.org/ 10.1126/sciadv. 1701138

S.N. Raymond, T. Quinn, J.I. Lunine, Making other earths: dynamical simulations of terrestrial planet formation and water delivery. Icarus 168, 1-17 (2004). https://doi.org/10.1016/j.icarus.2003.11.019

S.N. Raymond, T. Quinn, J.I. Lunine, High-resolution simulations of the final assembly of Earth-like planets I. Terrestrial accretion and dynamics. Icarus 183, 265-282 (2006). https://doi.org/10.1016/j. icarus.2006.03.011

S.N. Raymond, T. Quinn, J.I. Lunine, High-resolution simulations of the final assembly of Earth-like planets. 2. Water delivery and planetary habitability. Astrobiology 7, 66-84 (2007). https://doi.org/ 10.1089/ast.2006.06-0126

S.N. Raymond, D.P. O’Brien, A. Morbidelli, N.A. Kaib, Building the terrestrial planets: constrained accretion in the inner Solar System. Icarus 203, 644-662 (2009). https://doi.org/10.1016/j.icarus.2009.05.016

S.N. Raymond, E. Kokubo, A. Morbidelli, R. Morishima, K.J. Walsh, Terrestrial planet formation at home and abroad, in Protostars and Planets VI, ed. by B. Beuther, R.S. Klessen, C.P. Dullemond, T. Henning (University of Arizona Press, Tucson, 2014), pp. 595-618 
S.N. Raymond, A. Izidoro, B. Bitsch, S.A. Jacobson, Did Jupiter's core form in the innermost parts of the Sun's protoplanetary disc? Mon. Not. R. Astron. Soc. 458, 2962-2972 (2016). https://doi.org/10.1093/ mnras/stw431

A. Reufer, M.M.M. Meier, W. Benz, R. Wieler, A hit-and-run giant impact scenario. Icarus 221, 296-299 (2012). https://doi.org/10.1016/j.icarus.2012.07.021

K. Righter, M.J. Drake, Effect of water on metal-silicate partitioning of siderophile elements: a high pressure and temperature terrestrial magma ocean and core formation. Earth Planet. Sci. Lett. 171, 383-399 (1999). https://doi.org/10.1016/S0012-821X(99)00156-9

K. Righter, D.P. O’Brien, Cosmochemistry Special feature: terrestrial planet formation. Proc. Natl. Acad. Sci. 108, 19165-19170 (2011). https://doi.org/10.1073/pnas.1013480108

A.S. Rivkin, J.P. Emery, Detection of ice and organics on an asteroidal surface. Nature 464, 1322-1323 (2010). https://doi.org/10.1038/nature09028

F. Robert, The D/H ratio in chondrites. Space Sci. Rev. 106, 87-101 (2003). https://doi.org/10.1023/A: 1024629402715

F. Robert, Solar system deuterium/hydrogen ratio, in Meteorites and the Early Solar System II, ed. by D.S. Lauretta, H.Y. McSween (University of Arizona Press, Tucson, 2006), pp. 341-351

D.C. Rubie, C.K. Gessmann, D.J. Frost, Partitioning of oxygen during core formation on the Earth and Mars. Nature 429, 58-61 (2004). https://doi.org/10.1038/nature02473

D.C. Rubie, D.J. Frost, U. Mann, Y. Asahara, F. Nimmo, K. Tsuno, P. Kegler, A. Holzheid, H. Palme, Heterogeneous accretion, composition and core-mantle differentiation of the Earth. Earth Planet. Sci. Lett. 301, 31-42 (2011). https://doi.org/10.1016/j.eps1.2010.11.030

D.C. Rubie, F. Nimmo, H.J. Melosh, Formation of the Earth's core, in Treatise on Geophysics, vol. 9, ed. by G. Schubert (Elsevier, Amsterdam, 2015a), pp. 43-79

D.C. Rubie, S.A. Jacobson, A. Morbidelli, D.P. O’Brien, E.D. Young, J. de Vries, F. Nimmo, H. Palme, D.J. Frost, Accretion and differentiation of the terrestrial planets with implications for the compositions of early-formed Solar System bodies and accretion of water. Icarus 248, 89-108 (2015b). https:// doi.org/10.1016/j.icarus.2014.10.015

J. Siebert, J. Badro, D. Antonangeli, F.J. Ryerson, Terrestrial accretion under oxidizing conditions. Science 339(6124), 1194-1197 (2013). https://doi.org/10.1126/science.1227923

M. Stimpfl, A.M. Walker, M.J. Drake, N.H. de Leeuw, P. Deymier, An ångström-sized window on the origin of water in the inner solar system: atomistic simulation of adsorption of water on olivine. J. Cryst. Growth 294, 83-95 (2006). https://doi.org/10.1016/j.jcrysgro.2006.05.057

C. Surville, L. Mayer, D.N.C. Lin, Dust capture and long-lived density enhancements triggered by vortices in 2D protoplanetary disks. Astrophys. J. 831, 82 (2016). https://doi.org/10.3847/0004-637X/831/1/82

F. Tera, D.A. Papanastassiou, G.J. Wasserburg, Isotopic evidence for a terminal lunar cataclysm. Earth Planet. Sci. Lett. 22, 1-21 (1974). https://doi.org/10.1016/0012-821X(74)90059-4

E. Thommes, M. Nagasawa, D.N.C. Lin, Dynamical shake-up of planetary systems. II. N-body simulations of solar system terrestrial planet formation induced by secular resonance sweeping. Astrophys. J. 676, 728-739 (2008). https://doi.org/10.1086/526408

M. Touboul, T. Kleine, B. Bourdon, H. Palme, R. Wieler, Late formation and prolonged differentiation of the Moon inferred from W isotopes in lunar metals. Nature 450, 1206-1209 (2007). https://doi.org/10. 1038/nature06428

K. Tsiganis, R. Gomes, A. Morbidelli, H.F. Levison, Origin of the orbital architecture of the giant planets of the Solar System. Nature 435, 459-461 (2005). https://doi.org/10.1038/nature03539

K.J. Walsh, A. Morbidelli, S.N. Raymond, D.P. O'Brien, A.M. Mandell, A low mass for Mars from Jupiter's early gas-driven migration. Nature 475, 206-209 (2011). https://doi.org/10.1038/nature10201

W.R. Ward, Density waves in the solar nebula-differential Lindblad torque. Icarus 67, 164-180 (1986). https://doi.org/10.1016/0019-1035(86)90182-X

W.R. Ward, On planetesimal formation: the role of collective particle behavior, in Origin of the Earth and Moon, ed. by R.M. Canup, K. Righter (University of Arizona Press, Tucson, 2000), pp. 75-84

S.J. Weidenschilling, Aerodynamics of solid bodies in the solar nebula. Mon. Not. R. Astron. Soc. 180, 57-70 (1977). https://doi.org/10.1093/mnras/180.2.57

S.J. Weidenschilling, Dust to planetesimals—settling and coagulation in the solar nebula. Icarus 44, 172-189 (1980). https://doi.org/10.1016/0019-1035(80)90064-0

S.J. Weidenschilling, Radial drift of particles in the solar nebula: implications for planetesimal formation. Icarus 165, 438-442 (2003). https://doi.org/10.1016/S0019-1035(03)00169-6

S.J. Weidenschilling, Initial sizes of planetesimals and accretion of the asteroids. Icarus 214, 671-684 (2011). https://doi.org/10.1016/j.icarus.2011.05.024

S.J. Weidenschilling, J.N. Cuzzi, Formation of planetesimals in the solar nebula, in Protostars and Planets III, ed. by E.H. Levy, J.I. Lunine (University of Arizona Press, Tucson, 1993), pp. 1031-1060 
S.J. Weidenschilling, D. Spaute, D.R. Davis, F. Marzari, K. Ohtsuki, Accretional evolution of a planetesimal swarm. Icarus 128, 429-455 (1997). https://doi.org/10.1006/icar.1997.5747

G.W. Wetherill, Radiometric chronology of the early solar system. Annu. Rev. Nucl. Part. Sci. 25, 283-328 (1975). https://doi.org/10.1146/annurev.ns.25.120175.001435

G.W. Wetherill, Why isn't Mars as big as Earth? in Lunar and Planetary Science Conference Abstracts, vol. 22 (1991), p. 1495

G.W. Wetherill, An alternative model for the formation of the asteroids. Icarus 100, 307-325 (1992). https:// doi.org/10.1016/0019-1035(92)90103-E

G.W. Wetherill, G.R. Stewart, Accumulation of a swarm of small planetesimals. Icarus 77, 330-357 (1989). https://doi.org/10.1016/0019-1035(89)90093-6

G.W. Wetherill, G.R. Stewart, Formation of planetary embryos-effects of fragmentation, low relative velocity, and independent variation of eccentricity and inclination. Icarus 106, 190 (1993). https://doi. org/10.1006/icar.1993.1166

B.J. Wood, J. Wade, M.R. Kilburn, Core formation and the oxidation state of the Earth: additional constraints from Nb, V and Cr partitioning. Geochim. Cosmochim. Acta 72, 1415-1426 (2008). https://doi.org/10. 1016/j.gca.2007.11.036

G. Wurm, J. Blum, J.E. Colwell, NOTE: a new mechanism relevant to the formation of planetesimals in the solar nebula. Icarus 151, 318-321 (2001). https://doi.org/10.1006/icar.2001.6620

A.N. Youdin, J. Goodman, Streaming instabilities in protoplanetary disks. Astrophys. J. 620, 459-469 (2005). https://doi.org/10.1086/426895

A.N. Youdin, F.H. Shu, Planetesimal formation by gravitational instability. Astrophys. J. 580, 494-505 (2002). https://doi.org/10.1086/343109

H. Zhang, J.L. Zhou, On the orbital evolution of a giant planet pair embedded in a gaseous disk. II. A Saturn-Jupiter configuration. Astrophys. J. 719, 671-684 (2010). https://doi.org/10.1088/0004-637X/ $719 / 1 / 671$ 